TRANSACTIONS OF THE

AMERICAN MATHEMATICAL SOCIETY

Volume 359, Number 6, June 2007, Pages 2915-2944

S 0002-9947(07)04166-9

Article electronically published on January 4, 2007

\title{
LINEAR RELATIONS IN THE CALKIN ALGEBRA FOR COMPOSITION OPERATORS
}

\author{
THOMAS KRIETE AND JENNIFER MOORHOUSE
}

\begin{abstract}
We consider this and related questions: When is a finite linear combination of composition operators, acting on the Hardy space or the standard weighted Bergman spaces on the unit disk, a compact operator?
\end{abstract}

\section{INTRODUCTION}

For $\beta \geq 1$, let $\mathcal{D}_{\beta}$ denote the reproducing kernel Hilbert space of functions analytic in the unit disk $D=\{z:|z|<1\}$ and having the kernel functions $k_{w}(z)=$ $(1-\bar{w} z)^{-\beta}$. Thus, $f(w)=\left\langle f, k_{w}\right\rangle$ for $w$ in $D$ and $f$ in $\mathcal{D}_{\beta}$. The Hardy space $H^{2}$ is exactly $\mathcal{D}_{1}$, and when $\beta>1, \mathcal{D}_{\beta}$ is the standard weighted Bergman space $A_{\alpha}^{2}$ with $\alpha+2=\beta$; see Section 2.1. We consider composition operators $C_{\varphi}: f \rightarrow f \circ \varphi$ acting on $\mathcal{D}_{\beta}$, where $\varphi$ is an analytic self-map of $D$. When $\beta \geq 1$, every $C_{\varphi}$ lies in $\mathcal{B}\left(\mathcal{D}_{\beta}\right)$, the algebra of bounded operators on $\mathcal{D}_{\beta}$. Unlike the classes of Toeplitz and Hankel operators which act on $\mathcal{D}_{\beta}$, the set of composition operators in $\mathcal{B}\left(\mathcal{D}_{\beta}\right)$ has no obvious additive or linear structure. However, in the Bergman space case $\beta>1$, the second author observed additive structure modulo the ideal $\mathcal{K}$ of compact operators and characterized those pairs $\varphi$ and $\psi$ for which $C_{\varphi}-C_{\psi}$ is compact [16]. Our purpose here is twofold: to present some analogous results for the $H^{2}$ case $\beta=1$, and to pass from additive to linear structure in the Calkin algebra $\mathcal{B}\left(\mathcal{D}_{\beta}\right) / \mathcal{K}$.

Composition operators which are themselves compact were characterized in the $A_{\alpha}^{2}$ case by MacCluer and Shapiro [15] and on $H^{2}$ by Shapiro [25]; in [22] Sarason found a different condition, sufficient for $H^{2}$ and necessary and sufficient for $L^{1}$, later shown by Shapiro and Sundberg [27] to be necessary in the $H^{2}$ case as well. The problem of compact difference was raised in an explicit form by Shapiro and Sundberg [26 and MacCluer [13; these authors found several criteria, some necessary and some sufficient. More recently, MacCluer, Ohno, and Zhao [14 have shown that compactness of $C_{\varphi}-C_{\psi}$ acting on $H^{\infty}$, the space of bounded analytic functions on $D$, is characterized in terms of the quantity $\rho(z)=\left|\frac{\varphi(z)-\psi(z)}{1-\overline{\varphi(z)} \psi(z)}\right|$, the pseudo-hyperbolic distance between values of $\varphi$ and $\psi$. The second author of the present article subsequently found that $\rho$ plays a related role on the Bergman spaces, as follows.

Received by the editors July 14, 2004 and, in revised form, July 7, 2005.

2000 Mathematics Subject Classification. Primary 47B33; Secondary 47B32.

Key words and phrases. Composition operator, Hardy space, Bergman spaces, linear relations modulo the compact operators.

Work of the first author was supported in part by a sesquicentennial associateship at the University of Virginia.

(C)2007 American Mathematical Society Reverts to public domain 28 years from publication 
Theorem A ([16]). Suppose that $\varphi$ and $\psi$ are analytic self-maps of $D$ and $\alpha>-1$. Then $C_{\varphi}-C_{\psi}$ is a compact operator on $A_{\alpha}^{2}$ if and only if

$$
\lim _{|z| \rightarrow 1} \rho(z)\left\{\frac{1-|z|^{2}}{1-|\varphi(z)|^{2}}+\frac{1-|z|^{2}}{1-|\psi(z)|^{2}}\right\}=0 .
$$

Let $F(\varphi)$ denote the set of points in the unit circle $\partial D$ at which $\varphi$ has a finite angular derivative in the sense of Caratheodory; see Section 2.2. Also, we will use the notation $A \equiv B(\bmod \mathcal{K})$ to indicate that two bounded operators $A$ and $B$ have a compact difference. There is a "sum theorem," as follows:

Theorem B ([16]). Let $\varphi, \varphi_{1}, \ldots, \varphi_{n}$ be analytic self-maps of $D$ for which the sets $F\left(\varphi_{i}\right), i=1, \ldots, n$, are pairwise disjoint and with $F(\varphi)=F\left(\varphi_{1}\right) \cup \cdots \cup F\left(\varphi_{n}\right)$. Consider $C_{\varphi}, C_{\varphi_{1}}, \ldots, C_{\varphi_{n}}$ as acting on $A_{\alpha}^{2}$ where $\alpha>-1$, and let

$$
\rho_{i}=\left|\frac{\varphi-\varphi_{i}}{1-\bar{\varphi} \varphi_{i}}\right|, \quad i=1, \ldots, n .
$$

Then the following are equivalent:

(i) For each $i=1, \ldots, n$ and each $\zeta$ in $F\left(\varphi_{i}\right)$,

$$
\lim _{z \rightarrow \zeta} \rho_{i}(z)\left\{\frac{1-|z|^{2}}{1-|\varphi(z)|^{2}}+\frac{1-|z|^{2}}{1-\left|\varphi_{i}(z)\right|^{2}}\right\}=0 .
$$

(ii) $C_{\varphi} \equiv C_{\varphi_{1}}+\cdots+C_{\varphi_{n}}(\bmod \mathcal{K})$.

The following useful modification of Theorem A, which localizes the notion of compact difference, is implicit in [16].

Theorem C. Suppose that $\varphi$ and $\psi$ are analytic self-maps of $D, \alpha>-1$ and $G$ is a measurable subset of $D$. If

$$
\lim _{|z| \rightarrow 1} \chi_{G}(z) \rho(z)\left\{\frac{1-|z|^{2}}{1-|\varphi(z)|^{2}}+\frac{1-|z|^{2}}{1-|\psi(z)|^{2}}\right\}=0,
$$

then $M_{\chi_{G}}\left(C_{\varphi}-C_{\psi}\right)$ is a compact operator from $A_{\alpha}^{2}$ into its containing $L^{2}$ space. Here $M_{\chi_{G}}$ denotes the operator of multiplication by the characteristic function $\chi_{G}$.

Other recent related work includes the following: Bourdon, Levi, Narayan, and J. H. Shapiro 4] show that a composition operator associated with an "almost linear fractional" map is, in fact, a compact perturbation of a linear fractional composition operator; Bourdon [3] treats the question of compact difference vs. topological connectedness for linear fractional maps (see Section 6 below); J. E. Shapiro 24] shows that if $C_{\varphi}-C_{\psi}$ is compact on $H^{2}$, the singular parts of the Clark measures of $\varphi$ and $\psi$ coincide (see Section 2.3). Most recently, Nieminen and Saksman [19] have shown that the just-quoted condition of J. E. Shapiro on singular parts, plus uniform integrability of the differences of the absolutely continuous densities of the respective Clark measures of $\varphi$ and $\psi$, are together equivalent to compactness of $C_{\varphi}-C_{\psi}$ on $L^{1}$ (or, on the space of complex Borel measures on $\partial \mathbb{D}$ ) and are thus sufficient on $H^{2}$. In a different direction, Gorkin and Mortini [10 have characterized compactness for finite linear combinations of composition operators acting on uniform algebras.

Our analogues of Theorems A, B, and C for $H^{2}$ require some different methods. For us the key is an application of Clark measures, following ideas of Sarason [22, Cima and Matheson [5] and J. E. Shapiro [24]. In Section 3 we obtain essential 
norm estimates for weighted composition operators on $\mathrm{H}^{2}$ analogous to the CimaMatheson essential norm formula for (unweighted) composition operators [5]. We combine these results in Section 4 with a general principle of Moorhouse and Toews [17] and Carleson measure estimates as in [16] to obtain our $H^{2}$ results.

Section 5 is devoted to the question of when a given finite linear combination of composition operators is compact. We look at lower bounds, given in terms of firstand higher-order boundary data, for the essential norm of a linear combination; these results further develop ideas of MacCluer 13. We introduce the class $\mathcal{S}$ of analytic self-maps $\varphi$ of $D$ having "sufficient data" at every point in $\partial D$ where $\varphi$ makes significant contact with the boundary. For $\varphi$ and $\psi$ in $\mathcal{S}$ we make clear the obstructions to the essential norm $\left\|C_{\varphi}-C_{\psi}\right\|_{e}$ being small, the conditions under which it must be small, and when it is zero, that is, when $C_{\varphi}-C_{\psi}$ is compact. For $\varphi_{1}, \ldots, \varphi_{n}$ in $\mathcal{S}$, we characterize, via a finite system of linear equations involving boundary data of these maps, those coefficients for which $c_{1} C_{\varphi_{1}}+\cdots+c_{n} C_{\varphi_{n}}$ is compact. An application is a simple algorithm for determining the dimension of the vector space in $\mathcal{B}\left(\mathcal{D}_{\beta}\right) / \mathcal{K}$ spanned by the cosets $\left[C_{\varphi_{1}}\right], \ldots,\left[C_{\varphi_{n}}\right]$.

The final Section 6 concerns a problem first studied by Berkson [2], subsequently considered by MacCluer [13, Shapiro and Sundberg [26], and most recently by Toews and the second author [17] and Bourdon [3]: to characterize those pairs $\varphi$ and $\psi$ for which $C_{\varphi}$ and $C_{\psi}$ lie in the same connected component of the topological space of composition operators, equipped with the norm topology on $\mathcal{B}\left(\mathcal{D}_{\beta}\right)$. We observe that a general sufficient condition of the second author for the Bergman space case [16] extends to $H^{2}$ and apply this result to those $C_{\varphi}$ with $\varphi$ lying in a certain subclass $\mathcal{S}_{0}$ of $\mathcal{S}$ to determine when $C_{\varphi}$ and $C_{\psi}$ lie in the same component of $\left\{C_{\varphi}: \varphi \in \mathcal{S}_{0}\right\}$.

A variation on ideas of Berkson [2], Shapiro and Sundberg [26], and MacCluer [13] (see Exercise 9.3.2 in [8]) states that if $\varphi_{1}, \ldots, \varphi_{n}$ are distinct analytic self-maps of $D$, and if $J(\varphi)$ denotes the set of points $e^{i \theta}$ in $\partial D$ with $\left|\varphi\left(e^{i \theta}\right)\right|=1$, then

$$
\left\|\sum_{j=1}^{n} c_{j} C_{\varphi_{j}}\right\|_{e}^{2} \geq \frac{1}{2 \pi} \sum_{j=1}^{n}\left|c_{j}\right|^{2}\left|J\left(\varphi_{i}\right)\right|,
$$

where $|J(\varphi)|$ is the arclength measure of $J(\varphi)$. Accordingly, to study the questions discussed above, we assume throughout that our analytic self-maps $\varphi$ of $D$ satisfy $\left|\varphi\left(e^{i \theta}\right)\right|<1$ a.e.

\section{Preliminaries}

Here we collect some preliminary facts used in the sequel.

2.1. The Hardy and Bergman spaces. The Hardy space $H^{2}=\mathcal{D}_{1}$ is the set of all functions $f(z)=\sum_{n=0}^{\infty} a_{n} z^{n}$ analytic in $D$ with

$$
\|f\|^{2} \equiv \sum_{n=0}^{\infty}\left|a_{n}\right|^{2}<\infty .
$$

Given $f$ in $H^{2}$, the non-tangential limit $f\left(e^{i \theta}\right)=\lim _{\angle z \rightarrow e^{i \theta}} f(z)$ exists for $d \theta$-almost every $e^{i \theta}$ in $\partial D$. Moreover, the correspondence $f(z) \rightarrow f\left(e^{i \theta}\right)$ allows one to think of $H^{2}$ as the closed subspace of $L^{2}=L^{2}\left(\partial D, \frac{d \theta}{2 \pi}\right)$ with orthonormal basis $\left\{e^{i n \theta}\right\}_{n=0}^{\infty}$. 
For $\alpha>-1$, the Bergman space $A_{\alpha}^{2}$ is the set of functions $f$ analytic in $D$ with

$$
\|f\|^{2}=\frac{\alpha+1}{\pi} \int_{D}|f(z)|^{2}\left(1-|z|^{2}\right)^{\alpha} d A(z)<\infty,
$$

where $d A$ is a Lebesgue area measure on $D$. As mentioned above, $\mathcal{D}_{\beta}=A_{\beta-2}^{2}$ for $\beta>1$, with equality of norms.

For information about $H^{2}$ and $A_{\alpha}^{2}$, see [9] and $[8$.

2.2. Angular derivatives. Let $\varphi$ be an analytic self-map of $D$. Then $\varphi$ has a (finite) angular derivative at $\zeta$ in $\partial D$ provided $\varphi(\zeta)$, the non-tangential limit of $\varphi$ at $\zeta$, exists and has modulus one, and

$$
\varphi^{\prime}(\zeta) \equiv \lim _{\angle z \rightarrow \zeta} \frac{\varphi(z)-\varphi(\zeta)}{z-\zeta}
$$

exists as a finite complex number. If the angular derivative $\varphi^{\prime}(\zeta)$ fails to exist, we write $\left|\varphi^{\prime}(\zeta)\right|=\infty$. In either case the Julia-Caratheodory Theorem [8] asserts in part that

$$
\liminf _{z \rightarrow \zeta} \frac{1-|\varphi(z)|}{1-|z|}=\left|\varphi^{\prime}(\zeta)\right|
$$

where the limit inferior is taken unrestrictedly in $D$; moreover $\left|\varphi^{\prime}(\zeta)\right|>0$. Throughout we write $F(\varphi)$ for the set of all points in $\partial D$ where $\varphi$ has a finite angular derivative. For $\zeta$ in $F(\varphi)$ we have the relation $\varphi^{\prime}(\zeta)=\bar{\zeta} \varphi(\zeta)\left|\varphi^{\prime}(\zeta)\right|$. A condition necessary for the composition operator $C_{\varphi}$ to act compactly on $H^{2}$ is that $F(\varphi)$ be empty [28]. On the Bergman space $A_{\alpha}^{2}$, this condition is both necessary and sufficient [15].

2.3. Clark measures. Let $\varphi$ be an analytic self-map of $D$. If $|\alpha|=1$, there exists a finite positive Borel measure $\mu_{\alpha}$ on $\partial D$ such that

$$
\frac{1-|\varphi(z)|^{2}}{|\alpha-\varphi(z)|^{2}}=\operatorname{Re}\left(\frac{\alpha+\varphi(z)}{\alpha-\varphi(z)}\right)=\int_{\partial D} P_{z}\left(e^{i t}\right) d \mu_{\alpha}(t)
$$

for $z$ in $D$, where

$$
P_{z}\left(e^{i t}\right)=\frac{1-|z|^{2}}{\left|e^{i t}-z\right|^{2}}
$$

is the Poisson kernel at $z$. The existence of $\mu_{\alpha}$ follows since the left side of equation (2.1) is a positive harmonic function. The measures $\mu_{\alpha}$ (the Clark measures of $\varphi$ ) were introduced as an operator-theoretic tool by D. N. Clark [6], and have been further analyzed by Alexsandrov [1], Poltoratski [20], and Sarason [23].

On decomposing $\mu_{\alpha}=\mu_{\alpha}^{a c}+\mu_{\alpha}^{s}$, where $\mu_{\alpha}^{a c}$ and $\mu_{\alpha}^{s}$ are, respectively, the absolutely continuous and singular parts with respect to Lebesgue measure, one finds by Fatou's theorem [9] that

$$
\mu_{\alpha}^{a c}=\frac{1-\left|\varphi\left(e^{i \theta}\right)\right|^{2}}{\left|\alpha-\varphi\left(e^{i \theta}\right)\right|^{2}} \frac{d \theta}{2 \pi} .
$$

The singular part $\mu_{\alpha}^{s}$ is carried by $\varphi^{-1}(\{\alpha\})$, the set of those $\zeta$ in $\partial D$ where $\varphi(\zeta)$ exists and equals $\alpha$, and is itself the sum of the pure point measure

$$
\mu_{\alpha}^{p p}=\sum_{\varphi(\zeta)=\alpha} \frac{1}{\left|\varphi^{\prime}(\zeta)\right|} \delta_{\zeta}
$$


(here $\delta_{\zeta}$ is the unit point mass at $\zeta$ ) and a continuous singular measure $\mu_{\alpha}^{c s}$, either of which can vanish.

Let us write

$$
E(\varphi)=\overline{\bigcup_{|\alpha|=1} \operatorname{spt}\left(\mu_{\alpha}^{s}\right)}
$$

where $\operatorname{spt}(\mu)$ denotes the closed support of a measure $\mu$. It is clear from Eqn. (2.2) that $F(\varphi)$ is a subset of $E(\varphi)$.

2.4. Essential norms. Let $H$ and $L$ be separable Hilbert spaces and write $\mathcal{B}(H, L)$ for the space of bounded operators from $H$ to $L$. Let $\mathcal{K}$ denote the subspace of compact operators in $\mathcal{B}(H, L)$. The essential norm $\|T\|_{e}$ of an operator $T$ in $\mathcal{B}(H, L)$ is the operator-norm distance from $T$ to $\mathcal{K}$. We will find this alternate description useful:

$$
\|T\|_{e}=\sup _{\left\{f_{n}\right\} \in \mathcal{U}}\left(\limsup _{n \rightarrow \infty}\left\|T f_{n}\right\|\right)
$$

where $\mathcal{U}$ is the collection of all sequences $\left\{f_{n}\right\}$ of unit vectors in $H$ which tend to zero weakly.

2.5. Carleson measures. For a point $\zeta$ on the unit circle and $\delta>0$, let $S(\zeta, \delta)$ $=\{z \in D:|\zeta-z|<\delta\}$. If $\mu$ is a finite positive Borel measure on $D$ and $\beta \geq 1$, we consider the quantities

$$
\Delta_{\beta}(\mu)=\sup _{|\zeta|=1, \delta>0} \frac{\mu(S(\zeta, \delta))}{\delta^{\beta}}, \quad \Delta_{\beta}^{*}(\mu)=\limsup _{\delta \rightarrow 0}\left\{\sup _{|\zeta|=1} \frac{\mu(S(\zeta, \delta))}{\delta^{\beta}}\right\} .
$$

One often says $\mu$ is a $\beta$-Carleson measure if $\Delta_{\beta}(\mu)<\infty$, and a vanishing $\beta$-Carleson measure if $\Delta_{\beta}^{*}(\mu)=0$. The reader might consult 8 for the history of the following well-known result. The statement about $\|J\|_{e}$ can be deduced from ideas in the proof of Theorem 3.12 in 8 .

Theorem 2.1. Let $\mu$ be a finite positive Borel measure on $D$ and assume $\beta \geq 1$. Then:

(i) The space $\mathcal{D}_{\beta}$ (considered as a space of analytic functions on D) is contained in $L^{2}(\mu)$ if and only if $\mu$ is a $\beta$-Carleson measure. In this case the inclusion map $J: \mathcal{D}_{\beta} \rightarrow L^{2}(\mu)$ is bounded with norm comparable to $\sqrt{\Delta_{\beta}(\mu)}$.

(ii) If $\mathcal{D}_{\beta}$ is contained in $L^{2}(\mu)$, then $\|J\|_{e}$, the essential norm of the inclusion map, is comparable to $\sqrt{\Delta_{\beta}^{*}(\mu)}$. In particular, $J$ is compact if and only if $\mu$ is a vanishing $\beta$-Carleson measure.

2.6. A general scheme for compact difference and arc-connectedness. For a bounded analytic function $w$ on $\partial D$, one can form the associated multiplication operator $M_{w}: f \rightarrow w f$. If $\varphi$ is an analytic self-map of $D$, then we have the weighted composition operator $M_{w} C_{\varphi}$. Given two analytic self-maps of $D, \varphi$, and $\psi$, consider the self-maps $\varphi_{t}=t \varphi+(1-t) \psi, 0 \leq t \leq 1$. Based on the formal operator identity

$$
C_{\varphi_{s}}-C_{\varphi_{r}}=M_{\varphi-\psi}\left[\int_{r}^{s} C_{\varphi_{t}} d t\right] X
$$

$0 \leq r<s \leq 1$, and the fact that the differentiation operator $X=\frac{d}{d z}$ is a topological isomorphism of $H_{0}^{2}$ (the subspace of $H^{2}$ consisting of all functions that vanish at 
the origin) and the Bergman space $A_{1}^{2}$ (see [8]), the second author and C. Toews proved the following:

Theorem 2.2 ([17]). Let $\varphi, \psi$, and $\varphi_{t}, 0 \leq t \leq 1$, be as above.

(i) Suppose the weighted composition operators $M_{\varphi-\psi} C_{\varphi_{t}}$ act boundedly from $A_{1}^{2}$ to $H^{2}$, with uniformly bounded norms, $0 \leq t \leq 1$. Then, there is a constant $B>0$ such that, as operators on $H^{2}$,

$$
\left\|C_{\varphi_{s}}-C_{\varphi_{r}}\right\| \leq B|s-r|, 0 \leq r<s<1 .
$$

(ii) Suppose that $\varphi, \psi$, and $\varphi_{t}$ satisfy the hypotheses of part (i) above, and in addition, that for each $t, 0 \leq t \leq 1, M_{\varphi-\psi} C_{\varphi_{t}}$ is a compact operator from $A_{1}^{2}$ to $H^{2}$. Then $C_{\varphi}-C_{\psi}$ is a compact operator on $H^{2}$.

The above result remains true if one replaces $H^{2}$ and $A_{1}^{2}$ by $A_{\alpha}^{2}$ and $A_{\alpha+2}^{2}$, respectively, where $\alpha>-1$; see [17.

\section{Weighted COMPOSITION OPERATORS ON $H^{2}$ AND $L^{2}$}

For the analytic self-maps of $D$ considered here (those with $\left|\varphi\left(e^{i \theta}\right)\right|<1$ a.e. on $\partial D$ ), Sarason 22 found a convenient representation of $C_{\varphi}$ as an integral operator on $H^{2}$ and even on the larger space $L^{2}=L^{2}\left(\partial D, \frac{d \theta}{2 \pi}\right)$. For $f$ in $L^{2}$, extend $f$ to a harmonic function in $D$ via the Poisson integral: $f(z)=\int_{\partial D} P_{z}\left(e^{i t}\right) f\left(e^{i t}\right) \frac{d t}{2 \pi}$. Putting $\left(C_{\varphi} f\right)\left(e^{i \theta}\right)=f\left(\varphi\left(e^{i \theta}\right)\right)$ using the extended $f$ (since $\left|\varphi\left(e^{i \theta}\right)\right|<1$ a.e.), one has

$$
\left(C_{\varphi} f\right)\left(e^{i \theta}\right)=\int_{\partial D} \frac{1-\left|\varphi\left(e^{i \theta}\right)\right|^{2}}{\left|e^{i t}-\varphi\left(e^{i \theta}\right)\right|^{2}} f\left(e^{i t}\right) \frac{d t}{2 \pi} .
$$

Using the Schur test for boundedness of integral operators (stated below), Sarason showed that $C_{\varphi}$ is compact on $H^{2}$ if the Clark measures $\mu_{\alpha}$ of $\varphi$ are absolutely continuous for all $\alpha$ in $\partial D$; J. H. Shapiro and C. Sundberg [27] established the converse via function-theoretic methods. Subsequently Cima and Matheson [5] discovered the following expression for the essential norm of an arbitrary $C_{\varphi}$ acting on $H^{2}$ :

$$
\left\|C_{\varphi}\right\|_{e}^{2}=\sup _{|\alpha|=1} \mu_{\alpha}^{s}(\partial D),
$$

a formula foreshadowed by C. Cowen's inequalities for smooth $\varphi$ [7, p. 84].

Here we adapt the integral operator approach to investigate essential norms of weighted composition operators

$$
M_{w} C_{\varphi}: f \rightarrow w \cdot(f \circ \varphi) .
$$

We allow $w$ in $L^{\infty}$ and consider $M_{w} C_{\varphi}$ as mapping $L^{2}$ to $L^{2}, H^{2}$ to $L^{2}$ or (in the event that $w$ is in $\left.H^{\infty}\right), H^{2}$ to $H^{2}$. In all cases we have the following:

Theorem 3.1. Let $\varphi$ be an analytic self-map of $D$ with $\left|\varphi\left(e^{i \theta}\right)\right|<1$ a.e. and having Clark measures $\mu_{\alpha},|\alpha|=1$. Suppose $w$ is a bounded measurable function on $\partial D$ such that $|w|$ is continuous at every point of $E(\varphi)$. Then

$$
\sup _{|\alpha|=1} \int_{\partial D}|w|^{2} d \mu_{\alpha}^{s} \leq\left\|M_{w} C_{\varphi}\right\|_{e}^{2} \leq 4 \sup _{|\alpha|=1} \int_{\partial D}|w|^{2} d \mu_{\alpha}^{s} .
$$

In particular, $M_{w} C_{\varphi}$ is compact if and only if $w \equiv 0$ on $E(\varphi)$. 
For the proof, our essential tool is the following.

The Schur test [18, p. 282]. Consider two measure spaces $(X, \mu)$ and $(Y, \nu)$, and let $N$ be a measurable function on the product space $Y \times X$. Suppose there exist positive measurable functions $p$ on $X$ and $q$ on $Y$ and constants $A, B>0$ satisfying

$$
\begin{aligned}
\int_{X}|N(y, x)| p(x) d \mu(x) & \leq A q(y), y \text { in } Y, \\
\int_{Y}|N(y, x)| q(y) d \nu(y) & \leq B p(x), x \text { in } X .
\end{aligned}
$$

Then the formula

$$
(T f)(y)=\int_{X} N(y, x) f(x) d \mu(x)
$$

defines a bounded operator $T$ from $L^{2}(\mu)$ to $L^{2}(\nu)$ with $\|T\| \leq \sqrt{A B}$.

We will also need several lemmas. The first, and the final conclusion in the second, are due to J. E. Shapiro 24].

Lemma 3.2 (24). Let $\varphi$ be an analytic self-map of $D$ with Clark measures $\mu_{\alpha}$, $|\alpha|=1$. If $f$ is continuous on $\partial D$, then

$$
\lim _{r \nearrow 1} \int_{\partial D} f \frac{1-r^{2}}{|\alpha-r \varphi|^{2}} \frac{d \theta}{2 \pi}=\int_{\partial D} f d \mu_{\alpha}^{s}
$$

for $|\alpha|=1$.

Lemma 3.3. If $f$ is continuous on $\partial D$, then

$$
\lim _{r \nearrow 1} \int_{\partial D} f \frac{1-|r \varphi|^{2}}{|\alpha-r \varphi|^{2}} \frac{d \theta}{2 \pi}=\int_{\partial D} f d \mu_{\alpha}
$$

uniformly in $\alpha,|\alpha|=1$. Thus $\int_{\partial D} f d \mu_{\alpha}$ is a continuous function of $\alpha$.

Proof. For $0<r<1$, the function $\frac{1-|r \varphi|^{2}}{|\alpha-r \varphi|^{2}}$ is bounded and harmonic on $D$, and thus is the Poisson integral of its boundary function. It follows from this, and the definition of $\mu_{\alpha}$, that the conclusion holds for $f=P_{z}$, the Poisson kernel at any $z$ in $D$. Thus the conclusion holds when $f$ is a finite linear combination of Poisson kernels. Such finite linear combinations are uniformly dense in the continuous functions on $\partial D$, and the lemma follows.

The third lemma is a variant of exercise (7) in $\S 26$ of Halmos' treatise [11; for the proof the interested reader can easily adapt the hint given there.

Lemma 3.4. Let $f$ and $f_{n}$, for $n=1,2,3, \ldots$, be non-negative integrable functions on a measure space $(X, \mu)$ and suppose $\lambda \geq 0$. If $f_{n} \rightarrow f$, $\mu$-a.e. as $n \rightarrow \infty$, and

$$
\limsup _{n \rightarrow \infty}\left\{\int_{X} f_{n} d \mu-\int_{X} f d \mu\right\} \leq \lambda,
$$

then

$$
\limsup _{n \rightarrow \infty} \int_{X}\left|f_{n}-f\right| d \mu \leq \lambda .
$$

Proof of Theorem 3.1. We can write $w=|w| v$ where $v$ is measurable and unimodular on $\partial D$. Since $M_{w}=M_{v} M_{|w|}$ and $M_{v}$ is unitary, the theorem is unaltered by assuming that $w=|w| \geq 0$. For now we also assume that $w$ is continuous on $\partial D$. 
Consider the normalized kernel function

$$
K_{a}(z)=\frac{\sqrt{1-|a|^{2}}}{1-\bar{a} z}, \quad|a|<1 .
$$

If $|a| \rightarrow 1$, then $K_{a} \rightarrow 0$ weakly in $H^{2}$. Now take $a=r \alpha$ where $0 \leq r<1$ and $\alpha$ is fixed in $\partial D$. Then $\left|C_{\varphi} K_{r \alpha}\right|^{2}=\frac{1-r^{2}}{|\alpha-r \varphi|^{2}}$ and

$$
\int|w|^{2} d \mu_{\alpha}^{s}=\lim _{r \nearrow 1} \int|w|^{2} \frac{1-r^{2}}{|\alpha-r \varphi|^{2}} \frac{d \theta}{2 \pi}=\limsup _{r \nearrow 1}\left\|M_{w} C_{\varphi} K_{r \alpha}\right\|^{2}
$$

where Lemma 3.2 gives the first equality; the lower bound for $\left\|M_{w} C_{\varphi}\right\|_{e}^{2}$ follows from Eqn. (2.3). (Throughout the proof, all integrals are taken over $\partial D$.)

For the upper bound we consider $M_{w} C_{\varphi}$ as an integral operator from $L^{2}=$ $L^{2}\left(\partial D, \frac{d \theta}{2 \pi}\right)$ to $L^{2}\left(G, \frac{d \theta}{2 \pi}\right)$, where $G=\left\{e^{i \theta}: w\left(e^{i \theta}\right)>0\right\}$. By Eqn. (3.1) the kernel of this operator is

$$
K\left(e^{i \theta}, e^{i t}\right)=w\left(e^{i \theta}\right) \frac{1-\left|\varphi\left(e^{i \theta}\right)\right|^{2}}{\left|e^{i t}-\varphi\left(e^{i \theta}\right)\right|^{2}} .
$$

Similarly, if $0<r<1$, the kernel of the integral operator $M_{w} C_{r \varphi}$ is

$$
K_{r}\left(e^{i \theta}, e^{i t}\right)=w\left(e^{i \theta}\right) \frac{1-\left|r \varphi\left(e^{i \theta}\right)\right|^{2}}{\left|e^{i t}-r \varphi\left(e^{i \theta}\right)\right|^{2}} .
$$

Since $\|r \varphi\|_{\infty} \leq r<1, C_{r \varphi}$, and thus $M_{w} C_{r \varphi}$, are compact. We apply the Schur test to the integral operator $M_{w} C_{r \varphi}-M_{w} C_{\varphi}$, which has integral kernel $N=K_{r}-K$. We take $\mu=\frac{d \theta}{2 \pi}, \nu$ to be the restriction of $\frac{d \theta}{2 \pi}$ to $G, p\left(e^{i \theta}\right)=1$ and $q\left(e^{i \theta}\right)=w\left(e^{i \theta}\right)$. Then

$$
\begin{aligned}
\int\left|K_{r}\left(e^{i \theta}, e^{i t}\right)-K\left(e^{i \theta}, e^{i t}\right)\right| p\left(e^{i t}\right) \frac{d t}{2 \pi} \\
\leq w\left(e^{i \theta}\right) \int\left(\frac{1-\left|r \varphi\left(e^{i \theta}\right)\right|^{2}}{\left|e^{i t}-r \varphi\left(e^{i \theta}\right)\right|^{2}}+\frac{1-\left|\varphi\left(e^{i \theta}\right)\right|^{2}}{\left|e^{i t}-\varphi\left(e^{i \theta}\right)\right|^{2}}\right) \frac{d t}{2 \pi} \\
=2 w\left(e^{i \theta}\right)=2 q\left(e^{i \theta}\right),
\end{aligned}
$$

for all $e^{i \theta}$ in the circle. This is the first Schur hypothesis.

For the second Schur hypothesis, we write

$$
\lambda=\sup _{|\alpha|=1} \int|w|^{2} d \mu_{\alpha}^{s}
$$

and consider sequences $r_{n} \nearrow 1$ and $\left\{\alpha_{n}\right\}$ in $\partial D$. It is enough to show that

$$
\limsup _{n \rightarrow \infty} \int\left|K_{r_{n}}\left(e^{i \theta}, \alpha_{n}\right)-K\left(e^{i \theta}, \alpha_{n}\right)\right| w\left(e^{i \theta}\right) \frac{d \theta}{2 \pi} \leq 2 \lambda,
$$

for then, since $M_{w} C_{r_{n} \varphi}$ is compact, the Schur test with $A=2$ and $B=2 \lambda$ will imply that

as desired.

$$
\left\|M_{w} C_{\varphi}\right\|_{e}^{2} \leq \limsup _{n \rightarrow \infty}\left\|M_{w} C_{r_{n} \varphi}-M_{w} C_{\varphi}\right\|^{2} \leq 4 \lambda
$$

We may assume that $\alpha_{n}$ tends to some $\alpha$ in $\partial D$ as $n \rightarrow \infty$. First we use Lemma 3.4 with

$$
f_{n}=|w|^{2} \frac{1-|\varphi|^{2}}{\left|\alpha_{n}-\varphi\right|^{2}}, f=|w|^{2} \frac{1-|\varphi|^{2}}{|\alpha-\varphi|^{2}}
$$


Note that

$$
\int f \frac{d \theta}{2 \pi}=\int|w|^{2} d \mu_{\alpha}-\int|w|^{2} d \mu_{\alpha}^{s}
$$

and similarly for $f_{n}, \mu_{\alpha_{n}}$ and $\mu_{\alpha_{n}}^{s}$, so that

$$
\int\left(f_{n}-f\right) \frac{d \theta}{2 \pi}=\int|w|^{2} d \mu_{\alpha_{n}}-\int|w|^{2} d \mu_{\alpha}+\int|w|^{2} d \mu_{\alpha}^{s}-\int|w|^{2} d \mu_{\alpha_{n}}^{s} .
$$

By the final conclusion in Lemma 3.3, the difference of the first two terms tends to zero as $n \rightarrow \infty$, so that

$$
\limsup _{n \rightarrow \infty} \int\left(f_{n}-f\right) \frac{d \theta}{2 \pi} \leq \lambda .
$$

Since $f_{n} \rightarrow f$ a.e., Lemma 3.4 implies that

$$
\limsup _{n \rightarrow \infty} \int\left|K\left(e^{i \theta}, \alpha_{n}\right)-K\left(e^{i \theta}, \alpha\right)\right| w\left(e^{i \theta}\right) \frac{d \theta}{2 \pi} \leq \lambda .
$$

Now use Lemma 3.4 again, this time with

$$
f_{n}=|w|^{2} \frac{1-\left|r_{n} \varphi\right|^{2}}{\left|\alpha_{n}-r_{n} \varphi\right|^{2}}, f=|w|^{2} \frac{1-|\varphi|^{2}}{|\alpha-\varphi|^{2}} .
$$

We have

$$
\begin{aligned}
\int\left(f_{n}-f\right) \frac{d \theta}{2 \pi} & \leq\left.\left|\int\right| w\right|^{2} \frac{1-\left|r_{n} \varphi\right|^{2}}{\left|\alpha_{n}-r_{n} \varphi\right|^{2}} \frac{d \theta}{2 \pi}-\int|w|^{2} d \mu_{\alpha_{n}} \mid \\
& +\left.\left|\int\right| w\right|^{2} d \mu_{\alpha_{n}}-\int|w|^{2} d \mu_{\alpha} \mid \\
& +\left.\left|\int\right| w\right|^{2} d \mu_{\alpha}-\int|w|^{2} \frac{1-|\varphi|^{2}}{|\alpha-\varphi|^{2}} \frac{d \theta}{2 \pi} \mid .
\end{aligned}
$$

As $n \rightarrow \infty$, the first two terms on the right tend to zero by Lemma 3.3, while the last term is exactly $\int|w|^{2} d \mu_{\alpha}^{s}$, which does not exceed $\lambda$. Thus, according to Lemma 3.4 ,

$$
\limsup _{n \rightarrow \infty} \int\left|K_{r_{n}}\left(e^{i \theta}, \alpha_{n}\right)-K\left(e^{i \theta}, \alpha\right)\right| w\left(e^{i \theta}\right) \frac{d \theta}{2 \pi} \leq \lambda .
$$

Combining this with Eqn. (3.4) yields Eqn. (3.3) and thus the desired upper bound for $\left\|M_{w} C_{\varphi}\right\|_{e}^{2}$.

It remains to consider the case where $w=|w|$ is continuous at each point of $E(\varphi)$, but not necessarily on all of $\partial D$. Since $E(\varphi)$ is compact, there exists a function $g$ continuous on $\partial D$ with $g=w$ on $E(\varphi)$. Then $w-g$ is continuous at and vanishes at every point of $E(\varphi)$. Given $\epsilon>0$ one can construct a function $h$ continuous on $\partial D$ with $h=\epsilon$ on $E(\varphi)$ and $|w-g| \leq h$ on $\partial D$. Since $\left\|M_{w-g} C_{\varphi} f\right\| \leq\left\|M_{h} C_{\varphi} f\right\|$ for all $f$ in $L^{2},\left\|M_{w-g} C_{\varphi}\right\|_{e} \leq\left\|M_{h} C_{\varphi}\right\|_{e}$ by Eqn. (2.3). An application of this inequality and the triangle inequality yields

$$
\left\|M_{g} C_{\varphi}\right\|_{e}-\left\|M_{h} C_{\varphi}\right\|_{e} \leq\left\|M_{w} C_{\varphi}\right\|_{e} \leq\left\|M_{g} C_{\varphi}\right\|_{e}+\left\|M_{h} C_{\varphi}\right\|_{e} .
$$

Because $g$ and $h$ are both continuous on $\partial D$, we can apply our earlier argument to estimate $\left\|M_{g} C_{\varphi}\right\|_{e}$ and $\left\|M_{h} C_{\varphi}\right\|_{e}$; in particular, $\left\|M_{h} C_{\varphi}\right\|_{e}=O(\epsilon)$ as $\epsilon \rightarrow 0$. Since $\epsilon$ is arbitrary and $w=g$ on $E(\varphi)$, the theorem follows. 


\section{LOCAL COMPACT DIFFERENCE AND A SUM THEOREM FOR $H^{2}$}

Our plan for studying linear combinations in $\mathcal{B}\left(H^{2}\right) / \mathcal{K}$ is to decompose, mod $\mathcal{K}$, a composition operator into pieces associated to subsets of $E(\varphi)$ in a manner analogous to the decomposition in Theorem B above. This depends on an $H^{2}$ analogue of Theorem $\mathrm{C}$ above using Theorem 3.1 and $H^{2}$ versions of ideas from [16. Throughout, $\varphi$ and $\psi$ are analytic self-maps of $D$ with $|\varphi|<1$ a.e. and $|\psi|<1$ a.e. on $\partial D, \rho=\left|\frac{\varphi-\psi}{1-\bar{\varphi} \psi}\right|$, and $\varphi_{t}=t \varphi+(1-t) \psi, 0 \leq t \leq 1$. We require three lemmas.

Lemma 4.1. Let $a<1$ and suppose $G$ is a measurable subset of $\partial D$ with $\rho \leq a$ on $G$. Assume that $0 \leq t \leq 1$. Then $M_{\chi_{G}} M_{\varphi-\psi} C_{\varphi_{t}}$ acts boundedly from $A_{1}^{2}$ to $L^{2}$, and

$$
\left\|M_{\chi_{G}} M_{\varphi-\psi} C_{\varphi_{t}}\right\|_{\mathcal{B}\left(A_{1}^{2}, L^{2}\right)} \leq \frac{c}{1-a}\left\|M_{\chi_{G}} M_{\rho} C_{\varphi_{t}}\right\|_{\mathcal{B}\left(H^{2}, L^{2}\right)}
$$

where $c$ is an absolute constant. Moreover, the same inequality (with a different $c$ ) holds if both norms are replaced by the corresponding essential norms. In particular, if the operator $M_{\chi_{G}} M_{\rho} C_{\varphi_{t}}: H^{2} \rightarrow L^{2}$ is compact, so is $M_{\chi_{G}} M_{\varphi-\psi} C_{\varphi_{t}}: A_{1}^{2} \rightarrow L^{2}$.

Proof. We consider the measures

$$
\nu_{t}=\left(\chi_{G}|\varphi-\psi|^{2} \frac{d \theta}{2 \pi}\right) \circ \varphi_{t}^{-1}, \beta_{t}=\left(\chi_{G} \rho^{2} \frac{d \theta}{2 \pi}\right) \circ \varphi_{t}^{-1}
$$

on the disk $D$. As in [16] we have

$$
\begin{aligned}
\frac{1-\left|\varphi_{t}\right|^{2}}{|1-\bar{\varphi} \psi|} & =\left|1+\bar{\varphi} \frac{\left(\psi-\varphi_{t}\right)}{1-\bar{\varphi} \psi}+\varphi_{t} \frac{\left(\bar{\varphi}-\bar{\varphi}_{t}\right)}{1-\bar{\varphi} \psi}\right| \\
& =\left|1-t \bar{\varphi} \frac{(\varphi-\psi)}{1-\bar{\varphi} \psi}+(1-t) \varphi_{t} \frac{(\bar{\varphi}-\bar{\psi})}{1-\bar{\varphi} \psi}\right| \\
& \geq 1-\rho .
\end{aligned}
$$

Thus, if $\zeta$ lies in $\partial D, \delta>0$ and $S(\zeta, \delta)=\{z \in D:|z-\zeta|<\delta\}$, on the set $G \cap \varphi_{t}^{-1}(S(\zeta, \delta))$ we have

$$
|\varphi-\psi|^{2}=\rho^{2}|1-\bar{\varphi} \psi|^{2} \leq \rho^{2}\left(\frac{1-\left|\varphi_{t}\right|^{2}}{1-a}\right)^{2} \leq \frac{4 \delta^{2}}{(1-a)^{2}} \rho^{2} .
$$

It follows that

$$
\frac{\nu_{t}(S(\zeta, \delta))}{\delta^{3}} \leq \frac{4}{(1-a)^{2}} \frac{\beta_{t}(S(\zeta, \delta))}{\delta}
$$

so in the terminology of Section 2.5,

$$
\Delta_{3}\left(\nu_{t}\right) \leq \frac{4}{(1-a)^{2}} \Delta_{1}\left(\beta_{t}\right) \text { and } \Delta_{3}^{*}\left(\nu_{t}\right) \leq \frac{4}{(1-a)^{2}} \Delta_{1}^{*}\left(\beta_{t}\right)
$$

Since for all bounded analytic functions $h$ we have

$$
\int_{D}|h|^{2} d \nu_{t}=\left\|M_{\chi_{G}} M_{\varphi-\psi} C_{\varphi_{t}} h\right\|_{L^{2}}^{2} \text { and } \int_{D}|h|^{2} d \beta_{t}=\left\|M_{\chi_{G}} M_{\rho} C_{\varphi_{t}} h\right\|_{L^{2}}^{2},
$$

the desired conclusion follows from Theorem 2.1 and the formula (2.3). 
Lemma 4.2. Let $G$ be an open set in $\partial D$ on which $\rho$ is essentially bounded away from one, and assume that $E(\varphi) \cap E(\psi) \cap G$ is closed. Then $E\left(\varphi_{t}\right) \cap G$ is contained in $E(\varphi) \cap E(\psi) \cap G$ for $0<t<1$.

Proof. For $z$ in $D$ we have

$$
\frac{1-\left|\varphi_{t}(z)\right|}{1-|z|} \geq t \frac{1-|\varphi(z)|}{1-|z|}+(1-t) \frac{1-|\psi(z)|}{1-|z|} .
$$

On letting $z \rightarrow \zeta$ in $\partial D$, we see that if $\varphi_{t}$ has a finite angular derivative at $\zeta$, so do $\varphi$ and $\psi$, that is, $F\left(\varphi_{t}\right) \subset F(\varphi) \cap F(\psi)$. The opposite containment holds by linearity of the angular derivative and so $F\left(\varphi_{t}\right)=F(\varphi) \cap F(\psi)$.

Suppose that $I$ is an open arc whose closure lies in $G$ and does not intersect both $E(\varphi)$ and $E(\psi)$. Let $\mu_{\alpha, t}$ be a Clark measure for $\varphi_{t}$. The point masses of $\mu_{\alpha, t}$, if any, are carried by $F\left(\varphi_{t}\right) \subset E(\varphi) \cap E(\psi)$, so that $\mu_{\alpha, t}$ puts no mass at the endpoints of $I$. A theorem of J. E. Shapiro 24] then states that

$$
\mu_{\alpha, t}^{s}(I)=\lim _{r \nearrow 1} \int_{I} \frac{1-r^{2}}{\left|\alpha-r \varphi_{t}\right|^{2}} \frac{d \theta}{2 \pi},
$$

where $\mu_{\alpha, t}^{s}$ is the singular part of $\mu_{\alpha, t}$. It is enough to show that this quantity is zero, for then $\operatorname{spt}\left(\mu_{\alpha, t}^{s}\right)$ cannot intersect $I$, and consequently neither can $E\left(\varphi_{t}\right)$.

For some $a<1$ we have $\rho \leq a$ on $G$. Thus for $e^{i \theta}$ in $G, \psi\left(e^{i \theta}\right)$ lies in the closed pseudo-hyperbolic disk with pseudo-hyperbolic radius $a$ and pseudo-hyperbolic center $\varphi\left(e^{i \theta}\right)$. On noting the Euclidean center and radius of this (also Euclidean) disk [8, p. 44], one can verify that there is a positive constant $c$, depending only on $a<1$, such that

$$
\left|\alpha-r \varphi_{t}\left(e^{i \theta}\right)\right| \geq c \max \left\{\left|\alpha-r \varphi\left(e^{i \theta}\right)\right|,\left|\alpha-r \psi\left(e^{i \theta}\right)\right|\right\}
$$

for all $\alpha$ in $\partial D, e^{i \theta}$ in $G$ and $0<r<1$.

Given any $\zeta$ in the closure $\bar{I}$ of the above $\operatorname{arc} I$, there exists an open $\operatorname{arc} A(\zeta)$ in $\partial \mathbb{D}$ containing $\zeta$ with either $\overline{A(\zeta)} \cap E(\varphi)$ or $\overline{A(\zeta)} \cap E(\psi)$ empty. The open cover $\{A(\zeta): \zeta \in \bar{I}\}$ for $\bar{I}$ has a finite subcover $\left\{A_{1}, \ldots, A_{n}, B_{1}, \ldots, B_{m}\right\}$ such that all of the sets $\bar{A}_{i} \cap E(\varphi), i=1, \ldots, n$, and $\bar{B}_{j} \cap E(\psi), j=1, \ldots, m$, are empty; of course, this subcover could consist only of $A_{i}$ 's, or only of $B_{j}$ 's. By Eqn. (4.1) and the previous paragraph,

$$
\begin{aligned}
\mu_{\alpha, t}^{s}(I) & =\lim _{r \nearrow 1} \int_{I} \frac{1-r^{2}}{\left|\alpha-r \varphi_{t}\right|^{2}} \frac{d \theta}{2 \pi} \\
& \leq \lim _{r \nearrow 1} \frac{1}{c}\left[\sum_{i=1}^{n} \int_{A_{i}} \frac{1-r^{2}}{|\alpha-r \varphi|^{2}} \frac{d \theta}{2 \pi}+\sum_{j=1}^{m} \int_{B_{j}} \frac{1-r^{2}}{|\alpha-r \psi|^{2}} \frac{d \theta}{2 \pi}\right] \\
& =\frac{1}{c}\left[\sum_{i=1}^{n} \mu_{\alpha, 0}^{s}\left(A_{i}\right)+\sum_{j=1}^{m} \mu_{\alpha, 1}^{s}\left(B_{j}\right)\right] \\
& =0,
\end{aligned}
$$

as desired.

Our third lemma localizes Theorem 2.2. The proof is as in [17, but now uses the operator Eqn. (2.5) left-multiplied by $M_{\chi_{G}}$. 
Lemma 4.3. Let $G$ be a measurable subset of $\partial D$. Suppose that the weighted composition operators $M_{\chi_{G}} M_{\varphi-\psi} C_{\varphi_{t}}$ act boundedly from $A_{1}^{2}$ to $L^{2}$ with norms uniformly bounded for $0 \leq t \leq 1$. If $M_{\chi_{G}} M_{\varphi-\psi} C_{\varphi_{t}}$ is compact for $0 \leq t \leq 1$, then $M_{\chi_{G}}\left(C_{\varphi}-C_{\psi}\right)$ is compact from $H^{2}$ to $L^{2}$.

We can now state our local compact difference theorem.

Theorem 4.4. Let $U$ be an open subset of $\partial D$ whose boundary intersects neither $E(\varphi)$ nor $E(\psi)$, and with $E(\varphi) \cap U=E(\psi) \cap U$. Suppose that $\rho$ can be re-defined on a set of measure zero (if necessary), so that $\lim _{e^{i \theta} \rightarrow \zeta} \rho\left(e^{i \theta}\right)=\rho(\zeta)=0$ for every $\zeta$ in $E(\varphi) \cap E(\psi) \cap U$. Then $M_{\chi_{U}}\left(C_{\varphi}-C_{\psi}\right)$ is a compact operator from $H^{2}$ to $L^{2}$.

Proof. Since the intersection of $E(\varphi) \cap E(\psi)$ with $U$ must be compact, there is an open subset $G$ of $U$, containing this intersection and such that $\rho \leq \frac{1}{2}$ on $G$. We have

$$
M_{\chi_{U}}\left(C_{\varphi}-C_{\psi}\right)=M_{\chi_{G}}\left(C_{\varphi}-C_{\psi}\right)+M_{\chi_{U \backslash G}} C_{\varphi}-M_{\chi_{U \backslash G}} C_{\psi} .
$$

The last two operators on the right are compact by Theorem 3.1. According to Lemma 4.3, compactness of $M_{\chi_{G}}\left(C_{\varphi}-C_{\psi}\right)$ will follow if we can show that the operators $M_{\chi_{G}} M_{\varphi-\psi} C_{\varphi_{t}}$ map $A_{1}^{2}$ into $L^{2}$, are uniformly bounded in norm and are each compact. Lemma 4.1 gives uniform boundedness since

$$
\left\|C_{\varphi_{t}}\right\|_{\mathcal{B}\left(H^{2}\right)}^{2} \leq \frac{2}{1-\left|\varphi_{t}(0)\right|} \leq 2 \max \left\{\frac{1}{1-|\varphi(0)|}, \frac{1}{1-|\psi(0)|}\right\}
$$

(see [8]), while compactness follows from Lemmas 4.1, 4.2, and Theorem 3.1.

We close this section with a sum theorem for $H^{2}$. Here $\varphi, \varphi_{1}, \varphi_{2}, \ldots, \varphi_{n}$ are analytic self-maps of $D$ with $|\varphi|<1$ a.e. and $\left|\varphi_{i}\right|<1$ a.e. on $\partial D, i=1,2, \ldots, n$.

Theorem 4.5. Let $\varphi, \varphi_{1}, \ldots, \varphi_{n}$ be as above. Suppose that

(a) The sets $E\left(\varphi_{i}\right), i=1, \ldots, n$, are pairwise disjoint, and $E(\varphi)$ coincides with $E\left(\varphi_{1}\right) \cup \cdots \cup E\left(\varphi_{n}\right)$, and

(b) the functions $\rho_{i}=\left|\frac{\varphi-\varphi_{i}}{1-\bar{\varphi} \varphi_{i}}\right|$ can be altered on a set of measure zero in $\partial D$ (if necessary) to guarantee that $\lim _{e^{i \theta} \rightarrow \zeta} \rho_{i}\left(e^{i \theta}\right)=\rho_{i}(\zeta)=0$ for all $\zeta$ in $E\left(\varphi_{i}\right)$, $i=1, \ldots, n$.

Then, as operators on $H^{2}, C_{\varphi} \equiv C_{\varphi_{1}}+\cdots+C_{\varphi_{n}}(\bmod \mathcal{K})$.

Proof. Clearly, we can find pairwise disjoint open sets $U_{1}, \ldots, U_{n}$ in $\partial D$ such that $U_{i}$ contains $E\left(\varphi_{i}\right)$ and $\rho_{i} \leq \frac{1}{2}$ on $U_{i}, i=1, \ldots, n$. Let $G=\partial D \backslash \bigcup_{i=1}^{n} U_{i}$, so that 
$\partial D=G \cup U_{1} \cup \cdots \cup U_{n}$. For a measurable subset $B$ of $\partial D$, let us write $M_{B}$ for $M_{\chi_{B}}$. Then

$$
\begin{aligned}
C_{\varphi}-C_{\varphi_{1}}-C_{\varphi_{2}}-\cdots-C_{\varphi_{n}} \\
=\left(M_{G}+M_{U_{1}}+\cdots+M_{U_{n}}\right)\left(C_{\varphi}-C_{\varphi_{1}}-\cdots-C_{\varphi_{n}}\right) \\
=M_{G} C_{\varphi}-\sum_{i=1}^{n} M_{G} C_{\varphi_{i}} \\
+M_{U_{1}}\left(C_{\varphi}-C_{\varphi_{1}}\right)-\sum_{i \neq 1} M_{U_{1}} C_{\varphi_{i}} \\
+M_{U_{2}}\left(C_{\varphi}-C_{\varphi_{2}}\right)-\sum_{i \neq 2} M_{U_{2}} C_{\varphi_{i}} \\
+\quad \\
+M_{U_{n}}\left(C_{\varphi}-C_{\varphi_{n}}\right)-\sum_{i \neq n} M_{U_{n}} C_{\varphi_{i}}
\end{aligned}
$$

Theorem 3.1 tells us that every individual term on the right is compact except possibly $M_{U_{i}}\left(C_{\varphi}-C_{\varphi_{i}}\right), i=1, \ldots, n$. These, however, must be compact by Theorem 4.4 .

\section{Linear COMBinations MOD $\mathcal{K}$}

In this section we consider a class $\mathcal{S}$ of analytic self-maps of $D$ for which definitive computations can be done. For $\varphi_{1}, \ldots, \varphi_{n}$ in $\mathcal{S}$, we determine which linear combinations $c_{1} C_{\varphi_{1}}+\cdots+c_{n} C_{\varphi_{n}}$ are compact. We begin with some results which hold for arbitrary analytic self-maps of $D$.

5.1. A first-order lower bound for $\left\|c_{1} C_{\varphi_{1}}+\cdots+c_{n} C_{\varphi_{n}}\right\|_{e}$. Two analytic selfmaps of $D$ have the same first-order data at $\zeta$ in $\partial D$ provided $\zeta$ lies in both $F(\varphi)$ and $F(\psi), \varphi(\zeta)=\psi(\zeta)$ and $\varphi^{\prime}(\zeta)=\psi^{\prime}(\zeta)$. A special case of a theorem of MacCluer 113 states that if $C_{\varphi_{1}}, \ldots, C_{\varphi_{n}}$ act on $\mathcal{D}_{\beta}, \zeta$ is in $\partial D$, and no two of the maps $\varphi_{1}, \ldots, \varphi_{n}$ have the same first-order data at $\zeta$, then

$$
\left\|c_{1} C_{\varphi_{1}}+\cdots+c_{n} C_{\varphi_{n}}\right\|_{e}^{2} \geq \sum_{k=1}^{n}\left|c_{k}\right|^{2} \frac{1}{\left|\varphi_{k}^{\prime}(\zeta)\right|^{\beta}} .
$$

Here it is understood that if $\zeta$ is not in $F\left(\varphi_{k}\right)$, then $\left|\varphi_{k}^{\prime}(\zeta)\right|=\infty$. We will need a minor but useful extension of this result. For $\zeta$ in $\partial D$ and $M>0$, let $\Gamma_{\zeta, M}^{1}$ denote the curve in $D$ given by $\frac{|z-\zeta|}{1-|z|^{2}}=M$, the boundary of a non-tangential approach region with vertex at $\zeta$. At the point $\zeta$ the sides of this region make angle $\theta$ with the radius to $\zeta$, where $2 \cos \theta=\frac{1}{M}$. We will use the notation " $\lim _{\Gamma_{\zeta, M}^{1}}$ " to indicate a limit taken as $z \rightarrow \zeta$ along the starboard leg of $\Gamma_{\zeta, M}^{1}$ (that is, counterclockwise). 
Lemma 5.1. Suppose $\varphi$ and $\psi$ are analytic self-maps of D. Then

$$
\begin{aligned}
& \lim _{\Gamma_{\zeta, M}^{1}} \frac{1-|z|^{2}}{1-\overline{\varphi(z)} \psi(z)} \\
& \quad= \begin{cases}\overline{(1+i \tan \theta)}\left|\varphi^{\prime}(\zeta)\right|+(1+i \tan \theta)\left|\psi^{\prime}(\zeta)\right| & \text { if } \zeta \in F(\varphi) \cap F(\psi) \\
0 & \text { and } \varphi(\zeta)=\psi(\zeta),\end{cases} \\
& 0
\end{aligned}
$$

Proof. The Schwarz inequality applied to the $H^{2}$ kernel functions gives

$$
\frac{1-|z|^{2}}{|1-\overline{\varphi(z)} \psi(z)|} \leq\left(\frac{1-|z|^{2}}{1-|\varphi(z)|^{2}}\right)^{\frac{1}{2}}\left(\frac{1-|z|^{2}}{1-|\psi(z)|^{2}}\right)^{\frac{1}{2}} .
$$

Both factors on the right are bounded in $D$, and if $\zeta$ is not in $F(\varphi)$, say, the first factor tends to zero as $z \rightarrow \zeta$. Thus we may assume that $\zeta$ lies in $F(\varphi) \cap F(\psi)$. It is also clear that if $\varphi(\zeta) \neq \psi(\zeta)$, the left side of (5.1) tends to zero as $z \rightarrow \zeta$ along $\Gamma_{\zeta, M}^{1}$.

In the remaining case, $\zeta$ lies in $F(\varphi) \cap F(\psi)$ and $\varphi(\zeta)=\psi(\zeta)$. For $z$ in $\Gamma_{\zeta, M}^{1}$, we have

$$
\frac{1-\overline{\varphi(z)} \psi(z)}{1-|z|^{2}}=\frac{1-|\varphi(z)|^{2}}{1-|z|^{2}}+\overline{\varphi(z)} M \frac{\varphi(z)-\psi(z)}{|\zeta-z|} .
$$

Since $\Gamma_{\zeta, M}^{1}$ is nontangential at $\zeta$, the first term on the right tends to $\left|\varphi^{\prime}(\zeta)\right|$ as $z \rightarrow \zeta$ along $\Gamma_{\zeta, M}^{1}$. Moreover on the counterclockwise leg of $\Gamma_{\zeta, M}^{1}, \zeta-z \sim \zeta e^{i \theta}|\zeta-z|$ as $z \rightarrow$ $\zeta$. Recall that $\zeta \overline{\varphi(\zeta)} \varphi^{\prime}(\zeta)=\left|\varphi^{\prime}(\zeta)\right|$ and similarly for $\psi$. Since $M e^{i \theta}=\frac{1}{2}(1+i \tan \theta)$ and $\varphi(\zeta)=\psi(\zeta)$, we have

$$
\begin{aligned}
\lim _{\Gamma_{\zeta, M}^{1}} \frac{1-\overline{\varphi(z)} \psi(z)}{1-|z|^{2}} & =\left|\varphi^{\prime}(\zeta)\right|+M \zeta \overline{\varphi(\zeta)} e^{i \theta}\left(\psi^{\prime}(\zeta)-\varphi^{\prime}(\zeta)\right) \\
& =\frac{1}{2}\left[(1+i \tan \theta)\left|\psi^{\prime}(\zeta)\right|+\overline{(1+i \tan \theta)}\left|\varphi^{\prime}(\zeta)\right|\right],
\end{aligned}
$$

as desired.

For $\zeta$ in $F(\varphi)$ we call the vector $D_{1}(\varphi, \zeta)=\left(\varphi(\zeta), \varphi^{\prime}(\zeta)\right)$ the first-order data of $\varphi$ at $\zeta$. Suppose we fix analytic self-maps of $D, \varphi_{1}, \ldots, \varphi_{n}$. For $\zeta$ in $\partial D$, we denote by $\mathcal{D}_{1}(\zeta)$ the set of first-order data vectors at $\zeta$ associated to these self-maps:

$$
\mathcal{D}_{1}(\zeta)=\left\{D_{1}\left(\varphi_{j}, \zeta\right): 1 \leq j \leq n \text { and } \zeta \in F\left(\varphi_{j}\right)\right\} .
$$

Let us put $F=F\left(\varphi_{1}\right) \cup \cdots \cup F\left(\varphi_{n}\right)$.

Theorem 5.2. With the above notation, let $C_{\varphi_{1}}, \ldots, C_{\varphi_{n}}$ act on $\mathcal{D}_{\beta}$. Then for any complex numbers $c_{1}, \ldots, c_{n}$ and $\zeta$ in $\partial D$,

$$
\left\|c_{1} C_{\varphi_{1}}+\cdots+c_{n} C_{\varphi_{n}}\right\|_{e}^{2} \geq \sum_{\mathbf{d} \in \mathcal{D}_{1}(\zeta)}\left|\sum_{\substack{\zeta \in F\left(\varphi_{j}\right) \\ D_{1}\left(\varphi_{j}, \zeta\right)=\mathbf{d}}} c_{j}\right|^{2} \frac{1}{\left|d_{1}\right|^{\beta}}
$$

where $\mathbf{d}=\left(d_{0}, d_{1}\right)$. 
Proof. Referring to Lemma 5.1, we see that if $\varphi$ and $\psi$ are analytic self-maps of $D$, then $M$ tending to infinity means that $\theta \rightarrow \frac{\pi}{2}$, so that

$$
\lim _{M \rightarrow \infty} \lim _{\Gamma_{\zeta, M}^{1}} \frac{1-|z|^{2}}{1-\overline{\varphi(z)} \psi(z)}= \begin{cases}\frac{1}{\left|\varphi^{\prime}(\zeta)\right|} & \begin{array}{l}
\text { if } \zeta \in F(\varphi) \cap F(\psi) \text { and } \\
D_{1}(\varphi, \zeta)=D_{1}(\psi, \zeta),
\end{array} \\
0 & \text { otherwise. }\end{cases}
$$

As $|z| \rightarrow 1$ the normalized kernel functions $\frac{k_{z}}{\left\|k_{z}\right\|}$ tend weakly to zero in $\mathcal{D}_{\beta}$. Since $C_{\varphi}^{*} k_{z}=k_{\varphi(z)}$, we see from Eqn. (2.3) that

$$
\begin{aligned}
\left\|c_{1} C_{\varphi_{1}}+\cdots+c_{n} C_{\varphi_{n}}\right\|_{e}^{2} & \\
\geq & \lim _{M \rightarrow \infty} \lim _{\Gamma_{\zeta, M}^{1}}\left\|\left(\bar{c}_{1} C_{\varphi_{1}}^{*}+\cdots+\bar{c}_{n} C_{\varphi_{n}}^{*}\right) \frac{k_{z}}{\left\|k_{z}\right\|}\right\|_{e}^{2} \\
= & \sum_{j, \ell=1}^{n} \bar{c}_{j} c_{\ell} \lim _{M \rightarrow \infty} \lim _{\Gamma_{\zeta, M}^{1}}\left(\frac{1-|z|^{2}}{1-\overline{\varphi_{j}(z)} \varphi_{\ell}(z)}\right)^{\beta} \\
= & \sum_{\substack{\zeta \in F\left(\varphi_{j}\right) \cap F\left(\varphi_{\ell}\right) \\
D_{1}\left(\varphi_{j}, \zeta\right)=D_{1}\left(\varphi_{\ell}, \zeta\right)}} c_{j} \bar{c}_{\ell} \frac{1}{\left|\varphi_{j}(\zeta)\right|^{\beta}},
\end{aligned}
$$

which is a restatement of the desired conclusion.

Corollary 5.3. If $c_{1} C_{\varphi_{1}}+\cdots+c_{n} C_{\varphi_{n}}$ is compact on $\mathcal{D}_{\beta}$, then for every $\zeta$ in $F$ and every $\mathbf{d}$ in $\mathcal{D}_{1}(\zeta)$,

$$
\sum_{\substack{\zeta \in F\left(\varphi_{j}\right) \\ D_{1}\left(\varphi_{j}, \zeta\right)=\mathbf{d}}} c_{j}=0 .
$$

5.2. A remark on Theorem B. Corollary 5.3 shows that the hypothesis on angular derivative sets in Theorem B is actually implied by condition (ii) of the theorem.

Corollary 5.4. Suppose that $C_{\varphi}, C_{\varphi_{1}}, \ldots, C_{\varphi_{n}}$ act on $\mathcal{D}_{\beta}$ and

$$
C_{\varphi} \equiv C_{\varphi_{1}}+\cdots+C_{\varphi_{n}}(\bmod \mathcal{K}) .
$$

Then $F\left(\varphi_{1}\right), \ldots, F\left(\varphi_{n}\right)$ are pairwise disjoint and $F\left(\varphi_{1}\right) \cup \cdots \cup F\left(\varphi_{n}\right)$ coincides with $F(\varphi)$.

Proof. Let us write $\varphi=\varphi_{0}, c_{0}=1$, and $c_{j}=-1$ for $j=1, \ldots, n$, so that

$$
c_{0} C_{\varphi_{0}}+c_{1} C_{\varphi_{1}}+\cdots+c_{n} C_{\varphi_{n}} \in \mathcal{K} \text {. }
$$

By Corollary 5.3, each of the sets $\left\{j: \zeta \in F\left(\varphi_{j}\right)\right.$ and $\left.D_{1}\left(\varphi_{j}, \zeta\right)=\mathbf{d}\right\}$ is either empty or contains exactly two elements, namely zero and an integer from $\{1, \ldots, n\}$. The conclusion follows. 
5.3. Lower bounds from higher-order data. We have considered the first-order data $D_{1}(\varphi, \zeta)=\left(\varphi(\zeta), \varphi^{\prime}(\zeta)\right)$ for $\zeta$ in $F(\varphi)$. In what follows, we look at higher-order data vectors

$$
D_{k}(\varphi, \zeta)=\left(\varphi(\zeta), \varphi^{\prime}(\zeta), \varphi^{\prime \prime}(\zeta), \ldots, \varphi^{(k)}(\zeta)\right)
$$

at points where the corresponding derivatives make sense. Specifically, we say $\varphi$ has $k^{t h}$-order data at $\zeta$ in $\partial D$ if there exist complex numbers $b_{0}, b_{1}, \ldots, b_{k}$ with $\left|b_{0}\right|=1$ so that

$$
\varphi(z)=b_{0}+b_{1}(z-\zeta)+\cdots+b_{k}(z-\zeta)^{k}+o\left(|z-\zeta|^{k}\right)
$$

as $z \rightarrow \zeta$ unrestrictedly in $D$. In this case $\lim _{\angle z \rightarrow \zeta} \varphi^{(j)}(z)$ exists and equals $j ! b_{j}$ for $j=1, \ldots, n$ (see, for example, the argument on p. 47 in [23]); we refer to this limit as $\varphi^{(j)}(\zeta)$. Since $\left|b_{0}\right|=1, \zeta$ is in $F(\varphi)$ and $b_{1}$ is the angular derivative $\varphi^{\prime}(\zeta)$.

The model for this definition is of course a map which continues analytically across $\partial D$ near $\zeta$. Aside from the partial Taylor expansion we want our $\varphi$ to inherit another property of analyticity: order of contact. We say an analytic self-map $\varphi$ of $D$ has order of contact $c>0$ at $\zeta$ if $|\varphi(\zeta)|=1$ and

$$
\frac{1-\left|\varphi\left(e^{i \theta}\right)\right|^{2}}{\left|\varphi(\zeta)-\varphi\left(e^{i \theta}\right)\right|^{c}}
$$

is essentially bounded above and away from zero as $e^{i \theta} \rightarrow \zeta$. To clarify this and subsequent calculations, we map to the upper half-plane $\Omega=\{w: \operatorname{Im} w>0\}$. For any $\alpha$ in $\partial D$, consider the conformal map $\tau_{\alpha}(z)=i \frac{\alpha-z}{\alpha+z}$, which takes $D$ onto $\Omega$ and $\alpha$ to 0 . If $\varphi$ has a finite angular derivative at $\zeta$, then $u=\tau_{\varphi(\zeta)} \circ \varphi \circ \tau_{\zeta}^{-1}$ is an analytic self-map of $\Omega$ having a non-tangential limit $u(0)=0$ and a finite angular derivative $u^{\prime}(0)=\left|\varphi^{\prime}(\zeta)\right|$. Suppose for the moment that $\varphi$ has an analytic continuation to a neighborhood of $\zeta$, so the same is true of $u$ at the origin. For $w$ near zero, $u(w)=\sum_{n=1}^{\infty} a_{n} w^{n}$ with $a_{1}=u^{\prime}(0)$. If we assume $|\varphi|<1$ a.e. on $\partial D$, then

$$
\operatorname{Im} u(x)=\sum_{n=1}^{\infty}\left(\operatorname{Im} a_{n}\right) x^{n}
$$

is positive for real $x$ near (but not equal to) zero. The smallest natural number $n$ with $a_{n}$ non-real must be even, say $n=2 m$, and so $\operatorname{Im} u(x) \sim\left(\operatorname{Im} a_{2 m}\right) x^{2 m}$ as $x \rightarrow 0$; moreover, $\operatorname{Im} a_{2 m}>0$. It follows that near zero the image of $\mathbb{R}$ under $u$ is approximated by the curve $y=c x^{2 m}$ for appropriate $c>0$. Further, if $\tau_{\zeta}\left(e^{i \theta}\right)=x$, then

$$
\operatorname{Im} u(x)=\frac{1-\left|\varphi\left(e^{i \theta}\right)\right|^{2}}{\left|\varphi(\zeta)+\varphi\left(e^{i \theta}\right)\right|^{2}}
$$

and we find that $\frac{1-\left|\varphi\left(e^{i \theta}\right)\right|^{2}}{\left|\varphi(\zeta)-\varphi\left(e^{i \theta}\right)\right|^{2 m}}$ tends to a positive number as $e^{i \theta} \rightarrow \zeta$, so that $\varphi$ has order of contact $2 m$ at $\zeta$.

Definition 5.5. We say an analytic self-map $\varphi$ of $D$ has sufficient data at $\zeta$ in $\partial D$ if

(i) $\varphi$ has a finite angular derivative at $\zeta$;

(ii) $\varphi$ has an order of contact $2 m$ at $\zeta$ for some natural number $m$;

(iii) $\varphi$ has a $2 m^{\text {th }}$-order data at $\zeta$. 
If $\varphi$ has sufficient data at $\zeta$ with an order of contact $2 m$ and $u=\tau_{\varphi(\zeta)} \circ \varphi \circ \tau_{\zeta}^{-1}$ is as above, then $u$ has an analogous expansion at the origin,

$$
u(w)=\sum_{j=0}^{2 m} \frac{u^{(j)}(0)}{j !} w^{j}+o\left(|w|^{2 m}\right)
$$

here the derivatives can be realized as the non-tangential limits $\lim _{\angle w \rightarrow 0} u^{(j)}(w)$, $j=0,1, \ldots, 2 m$. Moreover, if $1 \leq k \leq 2 m$, the $k^{t h}$ order data vector $D_{k}(\varphi, \zeta)$ determines and is determined by the corresponding data $u^{\prime}(0), \ldots, u^{(k)}(0)$ of $u$ at zero.

Given $\zeta$ in $\partial D$, a natural number $k \geq 2$ and $M>0$, let $\Gamma_{\zeta, M}^{k}$ denote the locus of the equation $\frac{|\zeta-z|^{k}}{1-|z|^{2}}=M$ in $D$, a curve having "order of contact $k$ " with $\partial D$ at $\zeta$. We write " $\lim _{\Gamma_{\zeta, M}^{k}}$ " to indicate a limit taken as $z$ tends to $\zeta$ along $\Gamma_{\zeta, M}^{k}$.

Lemma 5.6. Suppose analytic self-maps of $D, \varphi$ and $\psi$, both have sufficient data at $\zeta$ in $\partial D$. Let $u=\tau_{\varphi(\zeta)} \circ \varphi \circ \tau_{\zeta}^{-1}$ and $v=\tau_{\psi(\zeta)} \circ \psi \circ \tau_{\zeta}^{-1}$, so that for $w$ near zero in $\Omega$,

$$
u(w)=\sum_{j=1}^{2 m} \frac{u^{(j)}(0)}{j !} w^{j}+o\left(|w|^{2 m}\right), \quad v(w)=\sum_{j=1}^{2 n} \frac{v^{(j)}(0)}{j !} w^{j}+o\left(|w|^{2 n}\right),
$$

where $2 m$ and $2 n$ are the respective orders of contact of $\varphi$ and $\psi$ at $\zeta$. Then, if $k \geq 2$ and $M>0$,

$$
\lim _{\Gamma_{\zeta, M}^{k}} \frac{1-|z|^{2}}{1-\overline{\varphi(z)} \psi(z)}=\left[\overline{\left(\frac{u^{\prime}(0)}{2}-i \frac{M}{2^{k-1} k !} u^{(k)}(0)\right)}+\left(\frac{v^{\prime}(0)}{2}-i \frac{M}{2^{k-1} k !} v^{(k)}(0)\right)\right]^{-1}
$$

provided $k \leq 2 m, k \leq 2 n$, and $D_{k-1}(\varphi, \zeta)=D_{k-1}(\psi, \zeta)$, while the limit is zero otherwise.

Proof. First we assume that $2 m \geq k, 2 n \geq k$ and $D_{k-1}(\varphi, \zeta)=D_{k-1}(\psi, \zeta)$. We put $\alpha=\varphi(\zeta)=\psi(\zeta)$ and observe from direct calculation that with $w=\tau_{\zeta}(z)$,

$$
\frac{v(w)-\overline{u(w)}}{2 i \operatorname{Im} w}=\frac{1-\overline{\varphi(z)} \psi(z)}{1-|z|^{2}} \cdot \frac{|\zeta+z|^{2}}{(\bar{\alpha}+\overline{\varphi(z)})(\alpha+\psi(z))}
$$

If we let $z \rightarrow \zeta$ along the curve $\Gamma_{\zeta, M}^{k}, w=\tau_{\zeta}(z)$ tends to zero along its image in $\Omega$, which is a slight enough perturbation of the curve $\tilde{\Gamma}_{k}$ defined by the equation $\frac{|w|^{k}}{\operatorname{Im} w}=\frac{M}{2^{k-2}}$ that the latter can be used to compute our limit. That is,

$$
\lim _{\Gamma_{\zeta, M}^{k}} \frac{1-\overline{\varphi(z)} \psi(z)}{1-|z|^{2}}=\lim _{\tilde{\Gamma}^{k}} \frac{v(w)-\overline{u(w)}}{2 i \operatorname{Im} w}
$$

Moreover,

$$
\frac{v(w)-\overline{u(w)}}{2 i \operatorname{Im} w}=\frac{\operatorname{Im} u(w)}{\operatorname{Im} w}+\frac{v(w)-u(w)}{2 i \operatorname{Im} w}
$$


We write

$$
u(w)=\sum_{j=1}^{2 m} a_{j} w^{j}+o\left(|w|^{2 m}\right), v(w)=\sum_{j=1}^{2 n} b_{j} w^{j}+o\left(|w|^{2 n}\right),
$$

and put $w=r e^{i \theta}$. Consider the first term on the right in Eqn. (5.2). Since $a_{1}, a_{2}, \ldots, a_{2 m-1}$ are real and $\operatorname{Im} a_{2 m}>0$, we have

$$
\begin{aligned}
\operatorname{Im} u(w) & =a_{1} r \sin \theta+a_{2} r^{2} \sin 2 \theta+\cdots+a_{2 m-1} r^{2 m-1} \sin (2 m-1) \theta \\
& +\left|a_{2 m}\right| r^{2 m} \sin \left(t_{2 m}+2 m \theta\right)+o\left(r^{2 m}\right),
\end{aligned}
$$

where $a_{2 m}=\left|a_{2 m}\right| e^{i t_{2 m}}$ with $0<t_{2 m}<\pi$. We take $w$ in $\tilde{\Gamma}^{k}$ and divide by $\operatorname{Im} w=r \sin \theta=\frac{2^{k-2}}{M} r^{k}$ to see that

$$
\lim _{\tilde{\Gamma}_{k}} \frac{\operatorname{Im} u(w)}{\operatorname{Im} w}=a_{1}+\left(\operatorname{Im} a_{k}\right) \frac{M}{2^{k-2}}, \quad 2 \leq k \leq 2 m,
$$

where we use the fact that $a_{k}$ is real if $k<2 m$.

Now consider the second term in Eqn. (5.2). Since $D_{k-1}(\varphi, \zeta)=D_{k-1}(\psi, \zeta)$, we have $a_{j}=b_{j}$ for $j<k$. Thus for $w$ in $\tilde{\Gamma}_{k}$,

$$
\frac{v(w)-u(w)}{2 i \operatorname{Im} w}=-i \frac{M}{2^{k-1}}\left(b_{k}-a_{k}\right) e^{i k \theta}+o(1) .
$$

Since $\theta \rightarrow 0$ as $w \rightarrow 0$ along $\tilde{\Gamma}_{k}$,

$$
\lim _{\tilde{\Gamma}_{k}} \frac{v(w)-u(w)}{2 i \operatorname{Im} w}=-i \frac{M}{2^{k-1}}\left(b_{k}-a_{k}\right) .
$$

This equation, Eqn. (5.3) and Eqn. (5.2) give the desired result.

Suppose now that $2 \leq k \leq \min \{2 m, 2 n\}$ and $D_{k-1}(\varphi, \zeta) \neq D_{k-1}(\psi, \zeta)$. Let $p$ be the smallest integer with $p \leq k-1$ and $a_{p} \neq b_{p}$. Then for $w=r e^{i \theta}$ in $\tilde{\Gamma}_{k}$,

$$
\frac{v(w)-u(w)}{2 i \operatorname{Im} w}=-i \frac{M}{2^{k-1}}\left(\frac{b_{p}-a_{p}}{r^{k-p}}\right) e^{i p \theta}+o\left(\frac{1}{r^{k-p}}\right),
$$

a quantity whose modulus tends to infinity as $w \rightarrow 0$ along $\tilde{\Gamma}_{k}$. Thus

$$
\lim _{\Gamma_{\zeta, M}^{k}} \frac{1-|z|^{2}}{1-\overline{\varphi(z)} \psi(z)}=0 .
$$

Finally, if $k>2 m$, Eqn. (5.4) follows from Eqn. (5.1), the definition of "order of contact" and simple estimates applied to the Clark measure inequality

$$
\frac{1-|\varphi(z)|^{2}}{|\varphi(\zeta)-\varphi(z)|^{2}} \geq \int_{\partial D} P_{z} \frac{1-|\varphi|^{2}}{|\varphi(\zeta)-\varphi|^{2}} \frac{d \theta}{2 \pi}
$$

The case $k>2 n$ is similar.

Now fix analytic self-maps $\varphi_{1}, \ldots, \varphi_{n}$ of $D$ and $\zeta$ in $F$. Assume that any $\varphi_{j}$ having finite angular derivative at $\zeta$ (that is, $\zeta$ is in $F\left(\varphi_{j}\right)$ ) in fact has sufficient data at $\zeta$. Given an integer $k \geq 2$, we write $\mathbb{M}_{k}(\zeta)$ for the set of those integers $j$, $1 \leq j \leq n$, for which $F\left(\varphi_{j}\right)$ contains $\zeta$ and the order of contact of $\varphi_{j}$ at $\zeta$ is at least $k$. Let us write $\mathcal{D}_{k}(\zeta)=\left\{D_{k}\left(\varphi_{j}, \zeta\right): j \in \mathbb{M}_{k}(\zeta)\right\}$. We have a higher-order analogue of Theorem 5.2. 
Theorem 5.7. Assume that $\varphi_{1}, \ldots, \varphi_{n}$ are analytic self-maps of $D$ as described above, and let $\zeta$ be in $F$. If $c_{1}, \ldots, c_{n}$ are complex, $k \geq 3$ and notation is as above,

$$
\left\|c_{1} C_{\varphi_{1}}+\cdots+c_{n} C_{\varphi_{n}}\right\|_{e}^{2} \geq \sum_{\mathbf{d} \in \mathcal{D}_{k-1}(\zeta)}\left|\sum_{\substack{j \in \mathbb{M}_{k}(\zeta) \\ D_{k-1}\left(\varphi_{j}, \zeta\right)=\mathbf{d}}} c_{j}\right|^{2} \frac{1}{\left|d_{1}\right|^{\beta}},
$$

where $\mathbf{d}=\left(d_{0}, d_{1}, \ldots, d_{k-1}\right)$ and each $C_{\varphi_{j}}$ acts on $\mathcal{D}_{\beta}$.

Proof. First consider analytic self-maps $\varphi$ and $\psi$ of $D$, each of which has sufficient data, with respective orders of contact $2 m$ and $2 n$, at a given $\zeta$ in $\partial D$. Let $u$ and $v$ be related to $\varphi$ and $\psi$ as in Lemma 5.6. Since $u^{(j)}(0)$ is real for $1 \leq j<2 m$ and $\operatorname{Im} u^{(2 m)}(0)>0$ (and similarly for $v^{(j)}(0)$ ), we see from Lemma 5.6 that

$$
\lim _{M \rightarrow \infty} \lim _{\Gamma_{\zeta, M}^{k-1}} \frac{1-|z|^{2}}{1-\overline{\varphi(z)} \psi(z)}=\left\{\begin{array}{cl}
\frac{1}{\left|\varphi^{\prime}(\zeta)\right|} \begin{array}{l}
\text { if } k \leq 2 m, k \leq 2 n, \text { and } \\
D_{k-1}(\varphi, \zeta)=D_{k-1}(\psi, \zeta),
\end{array} \\
0 \quad \text { otherwise. }
\end{array}\right.
$$

Proceeding as in the proof of Theorem 5.2, we find

$$
\begin{aligned}
\left\|c_{1} C_{\varphi_{1}}+\cdots+c_{n} C_{\varphi_{n}}\right\|_{e}^{2} \geq & \sum_{j, \ell=1}^{n} \overline{c_{j}} c_{\ell} \lim _{M \rightarrow \infty} \lim _{\Gamma_{\zeta, M}^{k-1}}\left(\frac{1-|z|^{2}}{1-\overline{\varphi_{j}(z)} \varphi_{\ell}(z)}\right)^{\beta} \\
= & \sum_{\substack{j, \ell \in \mathbb{M}_{k}(\zeta) \\
D_{k-1}\left(\varphi_{j}, \zeta\right)=D_{k-1}\left(\varphi_{\ell}, \zeta\right)}} \overline{c_{j}} c_{\ell} \frac{1}{\left|\varphi_{j}^{\prime}(\zeta)\right|^{\beta}},
\end{aligned}
$$

which is the desired conclusion.

The above theorem yields a higher-order version of MacCluer's lower bound for $\left\|C_{\varphi}-C_{\psi}\right\|_{e}$ in [13.

Corollary 5.8. Fix $\zeta$ in $\partial D$ and analytic self-maps $\varphi$ and $\psi$ of $D$, both of which have sufficient data at $\zeta$, with respective orders of contact $2 m$ and $2 n$.

(i) If $n<m$, then $\left\|C_{\varphi}-C_{\psi}\right\|_{e}^{2} \geq \frac{1}{\left|\varphi^{\prime}(\zeta)\right|^{\beta}}$.

(ii) If $n=m$ and $D_{2 m-1}(\varphi, \zeta) \neq D_{2 m-1}(\psi, \zeta)$, then

$$
\left\|C_{\varphi}-C_{\psi}\right\|_{e}^{2} \geq \frac{1}{\left|\varphi^{\prime}(\zeta)\right|^{\beta}}+\frac{1}{\left|\psi^{\prime}(\zeta)\right|^{\beta}} .
$$

Proof. Apply Theorem 5.7 with $\varphi_{1}=\varphi, \varphi_{2}=\psi, c_{1}=1$, and $c_{2}=-1$.

We need a more delicate version of Theorem 5.7 , which is conveniently expressed in terms of the following formalism. For $\beta \geq 1, \mathcal{D}_{\beta}^{+}$will denote the reproducing kernel Hilbert space of functions on the right half-plane $\Omega^{+}=\{z: \operatorname{Re} z>0\}$ having the kernel functions $k_{w}^{+}(z)=(z+\bar{w})^{-\beta}, w$ in $\Omega^{+}$. These spaces appear in the literature with various defining normalizations. When $\beta=1, \mathcal{D}_{\beta}^{+}$is the Hardy space on $\Omega^{+}$; see [12]. For $\beta>1, \mathcal{D}_{\beta}^{+}$is the weighted Bergman space of all functions $f$ analytic on $\Omega^{+}$for which

$$
\|f\|_{\mathcal{D}_{\beta}^{+}}^{2} \equiv \frac{2^{\beta-2}(\beta-1)}{\pi} \int_{\Omega^{+}}|f(x+i y)|^{2} x^{\beta-2} d x d y
$$


is finite; see [21, p. 74]. For us a key fact (used in Section 5.5) is this: $\left\{k_{w}^{+}: w \in \Omega_{+}\right\}$ is a linearly independent set in $\mathcal{D}_{\beta}^{+}$.

Now let $\mathbb{M}_{k}(\zeta)$ and $\mathcal{D}_{k}(\zeta)$ be as defined prior to Theorem 5.7.

Lemma 5.9. Let $\varphi_{1}, \ldots, \varphi_{n}$ be analytic self-maps of D. Fix $\zeta$ in $F$ and suppose that if $F\left(\varphi_{j}\right)$ contains $\zeta$, then $\varphi_{i}$ has sufficient data at $\zeta, j=1, \ldots, n$. Let $u_{j}$ be related to $\varphi_{j}$ as $u$ is related to $\varphi$ in Lemma 5.6. Then if $A>0$ and $k$ is an even natural number,

$$
\left\|c_{1} C_{\varphi_{1}}+\cdots+c_{n} C_{\varphi_{n}}\right\|_{e}^{2} \geq \sum_{\mathbf{d} \in \mathcal{D}_{k-1}(\zeta)}\left\|\sum_{\substack{j \in \mathbb{M}_{k}(\zeta) \\ D_{k-1}\left(\varphi_{j}, \zeta\right)=\mathbf{d}}} \overline{c_{j}} k_{\frac{u_{j}^{\prime}(0)}{2}-i A u_{j}^{(k)}(0)}^{+}\right\|_{\mathcal{D}_{\beta}^{+}}^{2} .
$$

Proof. We use Lemma 5.6 with $M=A 2^{k-1} k$ !. Then we have

$$
\begin{aligned}
& \left\|c_{1} C_{\varphi_{1}}+\cdots+c_{n} C_{\varphi_{n}}\right\|_{e}^{2} \\
& \geq \lim _{\Gamma_{\zeta, M}^{k}}\left\|\left(\overline{c_{1}} C_{\varphi_{1}}^{*}+\cdots+\overline{c_{n}} C_{\varphi_{n}}^{*}\right) \frac{k_{z}}{\left\|k_{z}\right\|}\right\|_{\mathcal{D}_{\beta}}^{2} \\
& \geq \lim _{\Gamma_{\zeta, M}^{k}} \sum \overline{c_{j}} c_{\ell}\left(\frac{1-|z|^{2}}{1-\overline{\varphi_{j}(z)} \varphi_{\ell}(z)}\right) \\
& =\sum_{D_{k-1}\left(\varphi_{j}, \zeta\right)=D_{k-1}\left(\varphi_{\ell}, \zeta\right)}^{\beta} \overline{c_{j} c_{\ell}}\left[\left(\frac{\overline{u_{j}^{\prime}(0)}}{2}-i A u_{j}^{(k)}(0)\right)+\left(\frac{u_{\ell}^{\prime}(0)}{2}-i A u_{\ell}^{(k)}(0)\right)\right]^{-\beta} \\
& =\sum_{\mathbf{d} \in \mathcal{D}_{k-1}(\zeta)}\left\|\sum_{\substack{j \in \mathbb{M}_{k}(\zeta) \\
D_{k-1}\left(\varphi_{j}, \zeta\right)=\mathbf{d}}} \overline{c_{j}} k_{\frac{u_{j}^{\prime}(0)}{2}-i A u_{j}^{(k)}(0)}^{+}\right\|_{\mathcal{D}_{\beta}^{+}}^{2}
\end{aligned}
$$

as desired. Note that since $u_{j}^{\prime}(0)>0, A>0$ and $\operatorname{Im} u_{j}^{(k)}(0) \geq 0$, the complex number $\frac{u_{j}(0)}{2}-i A u_{j}^{(k)}(0)$ lies in $\Omega^{+}$.

5.4. The class $\mathcal{S}$ and making $\left\|C_{\varphi}-C_{\psi}\right\|_{e}$ small. For maps $\varphi$ and $\psi$ with sufficient data at a given $\zeta$ in $\partial D$, Corollary 5.8 describes two obstructions to $\left\|C_{\varphi}-C_{\psi}\right\|_{e}$ being small:

(a) unequal orders of contact at $\zeta$;

(b) equal order of contact $2 m$ but $D_{2 m-1}(\varphi, \zeta) \neq D_{2 m-1}(\psi, \zeta)$.

In this section we estimate $\left\|C_{\varphi}-C_{\psi}\right\|_{e}$ in the absence of these obstructions and characterize when it is zero. We work within the class $\mathcal{S}$ of analytic self-maps $\varphi$ of $D$ for which $E(\varphi)$ is a finite set (so that $E(\varphi)=F(\varphi)$ ) and such that $\varphi$ has sufficient data at each point of $F(\varphi)$. For simplicity we restrict our attention to composition operators on $\mathcal{D}_{1}=H^{2}$. We write $\Omega$ for the upper half-plane $\{z: \operatorname{Im} z>0\}$; the 
pseudo-hyperbolic metric $\Lambda$ on $\Omega$ is given by

$$
\Lambda(z, w)=\left|\frac{z-w}{z-\bar{w}}\right| .
$$

Note that $0 \leq \Lambda<1$ on $\Omega \times \Omega$. Recall from Section 5.3 that if $\varphi$ has sufficient data at $\zeta$ in $F(\varphi)$ with order of contact $2 m$, and $u=\tau_{\varphi(\zeta)} \circ \varphi \circ \tau_{\zeta}^{-1}$, then $u^{(2 m)}(0)$ lies in $\Omega$.

Proposition 5.10. Fix $\zeta$ in $\partial D$ and suppose that $\varphi$ and $\psi$ have sufficient data with respective orders of contact $2 m$ and $2 n$ at $\zeta$, and moreover that $\varphi(\zeta)=\psi(\zeta)$. Write $\rho=\left|\frac{\varphi-\psi}{1-\bar{\varphi} \psi}\right|, u=\tau_{\varphi(\zeta)} \circ \varphi \circ \tau_{\zeta}^{-1}$ and $v=\tau_{\psi(\zeta)} \circ \psi \circ \tau_{\zeta}^{-1}$.

(a) If $2 m \neq 2 n$, or $2 m=2 n$ and $D_{2 m-1}(\varphi, \zeta) \neq D_{2 m-1}(\psi, \zeta)$, then $\rho\left(e^{i \theta}\right) \rightarrow 1$ as $e^{i \theta} \rightarrow \zeta$.

(b) If $2 m=2 n$ and $D_{2 m-1}(\psi, \zeta)=D_{2 m-1}(\varphi, \zeta)$, then

$$
\lim _{e^{i \theta \rightarrow \zeta}} \rho\left(e^{i \theta}\right)=\Lambda\left(u^{(2 m)}(0), v^{(2 m)}(0)\right)<1 .
$$

(c) $\rho\left(e^{i \theta}\right) \rightarrow 0$ as $e^{i \theta} \rightarrow \zeta$ if and only if $2 m=2 n$ and $D_{2 m}(\varphi, \zeta)=D_{2 m}(\psi, \zeta)$.

Proof. First assume that the hypotheses of (b) hold. Since $\psi(\zeta)=\varphi(\zeta)$, direct computation shows that if $\tau_{\zeta}\left(e^{i \theta}\right)=x$,

$$
\rho\left(e^{i \theta}\right)=\left|\frac{u(x)-v(x)}{u(x)-\overline{v(x)}}\right| .
$$

For $i=1,2, \ldots, 2 m-1$, the MacLaurin coefficient $a_{i}$ for $u$ is real and equal to the corresponding coefficient $b_{i}$ for $v$. On the other hand, $a_{2 m}=u^{(2 m)}(0) /(2 m)$ ! and $b_{2 m}=v^{(2 m)}(0) /(2 m)$ ! lie in $\Omega$. Clearly,

$$
\lim _{x \rightarrow 0}\left|\frac{u(x)-v(x)}{u(x)-\overline{v(x)}}\right|=\Lambda\left(a_{2 m}, b_{2 m}\right),
$$

which is the desired conclusion for (b).

Clearly (b) implies (c); the interested reader can easily verify (a).

For $\varphi, \psi$, and $\zeta$ as in Proposition 5.10, let us use the convention that $\rho(\zeta)=$ $\lim _{e^{i \theta} \rightarrow \zeta} \rho\left(e^{i \theta}\right)$. The limit exists by Proposition 5.10 and for $\varphi, \psi$ in $\mathcal{S}$ this convention, at worst, redefines $\rho$ on a finite set, leaving the multiplication operator $M_{\rho}$ unaltered. Note, however, that $\rho(\zeta)$ as just defined is in general not the same as the non-tangential $\operatorname{limit}_{\angle z \rightarrow \zeta} \rho(z)$.

Theorem 5.11. Suppose that $\varphi$ and $\psi$ are in $\mathcal{S}$ with $F(\varphi)=F(\psi)=F$, and that $C_{\varphi}$ and $C_{\psi}$ act on $H^{2}$. Suppose that at each $\zeta$ in $F, \varphi$ and $\psi$ have common order of contact $2 m(\zeta)$ with $D_{2 m(\zeta)-1}(\varphi, \zeta)=D_{2 m(\zeta)-1}(\psi, \zeta)$ and moreover that $\rho(\zeta) \leq \frac{1}{2}$. Then

$$
\frac{1}{4} \max _{\zeta \in F} \frac{1}{\left|\varphi^{\prime}(\zeta)\right|} \rho(\zeta)^{2} \leq\left\|C_{\varphi}-C_{\psi}\right\|_{e}^{2} \leq B \max _{\alpha \in \varphi(F)} \sum_{\varphi(\zeta)=\alpha} \frac{1}{\left|\varphi^{\prime}(\zeta)\right|} \rho(\zeta)^{2},
$$

where $B$ is an absolute constant and $\varphi(F)=\{\varphi(\zeta): \zeta \in F\}$. Moreover, the lower bound is valid without the assumption that $\rho(\zeta) \leq \frac{1}{2}, \zeta \in F$. 
Note. Suppose that $\varphi$ and $\psi$ satisfy the hypotheses of the theorem, and that $\mu_{\alpha}$ and $\nu_{\alpha}$ are their respective Clark measures. Then $\mu_{\alpha}^{s}$ and $\nu_{\alpha}^{s}$ are pure point, and since $\varphi$ and $\psi$ have the same first-order data,

$$
\nu_{\alpha}^{s}=\mu_{\alpha}^{s}=\sum_{\substack{\zeta \in F \\ \varphi(\zeta)=\alpha}} \frac{1}{\left|\varphi^{\prime}(\zeta)\right|} \delta_{\zeta} .
$$

The upper bound for $\left\|C_{\varphi}-C_{\psi}\right\|_{e}^{2}$ in the theorem then takes the form

$$
B \sup _{|\alpha|=1} \int \rho^{2} d \mu_{\alpha}^{s} .
$$

We expect that there is a lower bound of the same type.

Proof of Theorem 5.11. For $\zeta$ in $F$ write $u_{\zeta}=\tau_{\varphi(\zeta)} \circ \varphi \circ \tau_{\zeta}^{-1}$ and $v_{\zeta}=\tau_{\psi(\zeta)} \circ \psi \circ \tau_{\zeta}^{-1}$. According to Lemma 5.9, if $\zeta$ is in $F$ and $A>0$,

$$
\left\|C_{\varphi}-C_{\psi}\right\|_{e}^{2} \geq\left\|k_{\frac{u_{\zeta}^{\prime}(0)}{2}-i A u_{\zeta}^{(2 m(\zeta))}(0)}^{+}-k_{\frac{v_{\zeta}^{\prime}(0)}{2}-i A v_{\zeta}^{(2 m(\zeta))}(0)}^{+}\right\|_{H_{+}^{2}}^{2} .
$$

A calculation analogous to 8 , Lemma 9.11] shows that for $z, w$ in $\Omega_{+}$,

$$
\left\|k_{z}^{+}-k_{w}^{+}\right\|_{H_{+}^{2}}^{2}=\left|\frac{z-w}{z+\bar{w}}\right|^{2}\left(\frac{1}{z+\bar{z}}+\frac{1}{w+\bar{w}}\right) .
$$

We put

$$
\begin{gathered}
A=\frac{u_{\zeta}^{\prime}(0)}{\left|u_{\zeta}^{(2 m(\zeta))}(0)-\overline{v_{\zeta}^{(2 m(\zeta))}(0)}\right|}, \\
z=\frac{u_{\zeta}^{\prime}(0)}{2}-i A u_{\zeta}^{(2 m(\zeta))}(0), w=\frac{v_{\zeta}^{\prime}(0)}{2}-i A v_{\zeta}^{(2 m(\zeta))}(0),
\end{gathered}
$$

and note that $|z+\bar{w}| \leq 2 u_{\zeta}^{\prime}(0)$ while

$$
|z-w|=u_{\zeta}^{\prime}(0) \Lambda\left(u_{\zeta}^{(2 m(\zeta))}(0), v_{\zeta}^{(2 m(\zeta))}(0)\right) .
$$

It follows that

$$
\left\|k_{z}^{+}-k_{w}^{+}\right\|_{H_{+}^{2}}^{2} \geq \frac{1}{4 u_{\zeta}^{\prime}(0)} \Lambda\left(u_{\zeta}^{(2 m(\zeta))}(0), v_{\zeta}^{(2 m(\zeta))}(0)\right)^{2}
$$

which, by Proposition 5.10 and since $u_{\zeta}^{\prime}(0)=\left|\varphi^{\prime}(\zeta)\right|$, gives the lower bound for the essential norm of $C_{\varphi}-C_{\psi}$.

For the upper bound select a finite union $G$ of pairwise disjoint open arcs which contains $F$ and is such that $\rho \leq \frac{2}{3}$ on $G$. Now

$$
C_{\varphi}-C_{\psi}=M_{\chi_{G}}\left(C_{\varphi}-C_{\psi}\right)+M_{\chi_{\partial D \backslash G}} C_{\varphi}-M_{\chi_{\partial D \backslash G}} C_{\psi}
$$

and the last two terms on the right are compact operators by Theorem 3.1. Therefore $\left\|C_{\varphi}-C_{\psi}\right\|_{e}=\left\|M_{\chi_{G}}\left(C_{\varphi}-C_{\psi}\right)\right\|_{e}$ which we now estimate. To use Eqn. (2.3) it is permissible to restrict the sequence $\left\{f_{n}\right\}$ to a subspace of finite codimension. With notation as in Section 2.6, we pick a sequence of unit vectors $f_{n}$ in $H_{0}^{2}=(\text { ker } X)^{\perp}$ which converges weakly to zero. When applied to an $H^{2}$ function, the operator 
identity (2.5) holds pointwise in $D$ and thus, since the kernel functions span $H^{2}$, in the weak operator topology. Thus

$$
\left\|M_{\chi_{G}}\left(C_{\varphi}-C_{\psi}\right) f_{n}\right\|_{L^{2}} \leq\|X\|_{\mathcal{B}\left(H_{0}^{2}, A_{1}^{2}\right)} \int_{0}^{1}\left\|M_{\chi_{G}} M_{\varphi-\psi} C_{\varphi_{t}} g_{n}\right\|_{L^{2}} d t,
$$

where $g_{n}=X f_{n} /\left\|X f_{n}\right\|$. Since $X$ is bounded below on $H_{0}^{2}, g_{n} \rightarrow 0$ weakly in $A_{1}^{2}$. Now $\left\|M_{\chi_{G}} M_{\varphi-\psi} C_{\varphi_{t}}\right\|_{\mathcal{B}\left(A_{1}^{2}, L^{2}\right)}$ is bounded for $0 \leq t \leq 1$ by Lemma 4.1 and Eqn. (4.2), so we can apply Fatou's Lemma to conclude that

$$
\begin{aligned}
& \limsup _{n \rightarrow \infty}\left\|M_{\chi_{G}}\left(C_{\varphi}-C_{\psi}\right) f_{n}\right\|_{L^{2}} \\
& \quad \leq\|X\|_{B\left(H_{0}^{2}, A_{1}^{2}\right)} \int_{0}^{1} \limsup _{n \rightarrow \infty}\left\|M_{\chi_{G}} M_{\varphi-\psi} C_{\varphi_{t}} g_{n}\right\|_{L^{2}} d t .
\end{aligned}
$$

Moreover, by Eqn. (2.3) and Lemma 4.1 we have

$$
\begin{aligned}
\limsup _{n \rightarrow \infty}\left\|M_{\chi_{G}} M_{\varphi-\psi} C_{\varphi_{t}} g_{n}\right\|_{L^{2}} & \leq\left\|M_{\chi_{G}} M_{\varphi-\psi} C_{\varphi_{t}}\right\|_{e, \mathcal{B}\left(A_{1}^{2}, L^{2}\right)} \\
& \leq b\left\|M_{\chi_{G}} M_{\rho} C_{\varphi_{t}}\right\|_{e, \mathcal{B}\left(H^{2}, L^{2}\right)} \\
& \leq b\left\|M_{h} C_{\varphi_{t}}\right\|_{e, \mathcal{B}\left(H^{2}, L^{2}\right)},
\end{aligned}
$$

where $b>0, h$ is any continuous function on $\partial D$ with $h \geq \chi_{G} \rho$ on $\partial D$ and $h(\zeta)=\rho(\zeta)$ for $\zeta$ in $F$. Clearly we can choose such an $h$ while also requiring that $\rho \leq \frac{3}{4}$ on the set $W=\left\{e^{i \theta}: h\left(e^{i \theta}\right)>0\right\}$. Then, by Theorem 3.1 and Lemma 3.2, if $\mu_{\alpha, t}$ is a Clark measure for $\varphi_{t}$,

$$
\begin{aligned}
\left\|M_{h} C_{\varphi_{t}}\right\|_{e} & \leq 2 \sup _{|\alpha|=1}\left\{\int_{W} h^{2} d \mu_{\alpha, t}^{s}\right\}^{\frac{1}{2}} \\
& =2 \sup _{|\alpha|=1}\left\{\lim _{r \nearrow 1} \int_{W} h^{2} \frac{1-r^{2}}{\left|\alpha-r \varphi_{t}\right|^{2}} \frac{d \theta}{2 \pi}\right\}^{\frac{1}{2}} .
\end{aligned}
$$

Since $\rho \leq \frac{3}{4}$ on $W$ we see (as noted in the proof of Lemma 4.2) that there is a constant $c>0$ such that $\left|\alpha-r \varphi_{t}\left(e^{i \theta}\right)\right| \geq c\left|\alpha-r \varphi\left(e^{i \theta}\right)\right|$, for $\alpha$ in $\partial D, e^{i \theta}$ in $W$, $0<r<1$ and $0 \leq t<1$. Thus

$$
\begin{aligned}
\left\|M_{h} C_{\varphi_{t}}\right\|_{e} & \leq \frac{2}{c} \sup _{|\alpha|=1}\left\{\lim _{r \succ 1} \int_{\partial D} h^{2} \frac{1-r^{2}}{|\alpha-r \varphi|^{2}} \frac{d \theta}{2 \pi}\right\}^{\frac{1}{2}} \\
& =\frac{2}{c} \sup _{|\alpha|=1} \int_{\partial D} h^{2} d \mu_{\alpha}^{s},
\end{aligned}
$$

where $\left\{\mu_{\alpha}\right\}$ are the Clark measures for $\varphi$. Since each $\mu_{\alpha}^{s}$ is pure point in the present circumstances,

$$
\mu_{\alpha}^{s}= \begin{cases}\sum_{\substack{\zeta \in F \\ \varphi(\zeta)=\alpha}} \frac{1}{\left|\varphi^{\prime}(\zeta)\right|} \delta_{\zeta} & \text { if } \alpha \in \varphi(F), \\ 0 & \text { otherwise. }\end{cases}
$$

In view of Eqn. (2.3) and the arbitrariness of $\left\{f_{n}\right\}$ in $H_{0}^{2}$, this gives the desired upper bound for $\left\|C_{\varphi}-C_{\psi}\right\|_{e}$. 
Corollary 5.12. Suppose that $\varphi$ and $\psi$ lie in $\mathcal{S}$ with respective orders of contact $2 m(\zeta)$ and $2 n(\zeta)$ at each $\zeta$ in (respectively) $F(\varphi)$ and $F(\psi)$. Then the following are equivalent:

(i) $C_{\varphi}-C_{\psi}$ is compact on $H^{2}$.

(ii) $F(\psi)=F(\varphi)$ (we call this set $F$ ) and for all $\zeta$ in $F, 2 n(\zeta)=2 m(\zeta)$ and $D_{2 m(\zeta)}(\psi, \zeta)=D_{2 m(\zeta)}(\varphi, \zeta)$.

5.5. Linear relations $\bmod \mathcal{K}$. We fix $\varphi_{1}, \ldots, \varphi_{n}$ in $\mathcal{S}$ and again write $F$ for the union $F\left(\varphi_{1}\right) \cup \cdots \cup F\left(\varphi_{n}\right)$, a finite set. For $\zeta$ in $F$ and $k=2,4,6, \ldots$, let

$\mathbb{N}_{k}(\zeta)=\left\{j: F\left(\varphi_{j}\right)\right.$ contains $\zeta$ and $k$ is the order of contact of $\varphi_{j}$ at $\left.\zeta\right\}$.

We also write $\mathcal{E}_{k}(\zeta)=\left\{D_{k}\left(\varphi_{j}, \zeta\right): j\right.$ is in $\left.\mathbb{N}_{k}(\zeta)\right\}$.

Theorem 5.13. Let $\varphi_{1}, \ldots, \varphi_{n}$ be in $\mathcal{S}$ and set notation as above. Given complex numbers $c_{1}, \ldots, c_{n}$, the following are equivalent:

(i) $c_{1} C_{\varphi_{1}}+\cdots+c_{n} C_{\varphi_{n}}$ is compact on $\mathcal{D}_{\beta}$;

(ii) for every $\zeta$ in $F$, every even $k \geq 2$ and every $\mathbf{d}$ in $\mathcal{E}_{k}(\zeta)$,

$$
\sum_{\substack{j \in \mathbb{N}_{k}(\zeta) \\ D_{k}\left(\varphi_{j}, \zeta\right)=\mathbf{d}}} c_{j}=0
$$

Proof. First assume that $c_{1} C_{\varphi_{1}}+\cdots+c_{n} C_{\varphi_{n}}$ is compact. Fix $\zeta$ in $F$ and let $u_{j}$ be related to $\varphi_{j}$ as $u$ is related to $\varphi$ in Lemma 5.6. Let $\mathbb{M}_{k}(\zeta)$ and $\mathcal{D}_{k}(\zeta)$ be as defined prior to Theorem 5.7. According to Lemma 5.9 (with $A=1$ ), if $k \geq 2$, $\mathbf{d}$ is in $\mathcal{D}_{k-1}(\zeta)$, and $w_{j}=\frac{u_{j}^{\prime}(0)}{2}-i u_{j}^{(k)}(0)$, then

$$
\sum_{\substack{j \in \mathbb{M}_{k}(\zeta) \\ D_{k-1}\left(\varphi_{j}, \zeta\right)=\mathbf{d}}} \overline{c_{j}} k_{w_{j}}^{+}=0
$$

But if $D_{k-1}\left(\varphi_{j}, \zeta\right)$ and $D_{k-1}\left(\varphi_{\ell}, \zeta\right)$ coincide, $w_{j}=w_{\ell}$ exactly when $D_{k}\left(\varphi_{j}, \zeta\right)=$ $D_{k}\left(\varphi_{\ell}, \zeta\right)$. Using the fact that $\left\{k_{w}^{+}: \operatorname{Re} w>0\right\}$ is linearly independent in $\mathcal{D}_{\beta}^{+}$, we see that for every $\mathbf{d}$ in $\mathcal{D}_{k}(\zeta)$,

$$
\sum_{\substack{j \in \mathbb{M}_{k}(\zeta) \\ D_{k}\left(\varphi_{j}, \zeta\right)=\mathbf{d}}} c_{j}=0
$$

Since $\mathbb{M}_{k}(\zeta)$ is the union of the disjoint sets $\mathbb{N}_{k}(\zeta)$ and $\mathbb{M}_{k+1}(\zeta)$, we see from Eqn. (5.5) that

$$
\sum_{\substack{j \in \mathbb{N}_{k}(\zeta) \\ D_{k}\left(\varphi_{j}, \zeta\right)=\mathbf{d}}} c_{j}+\sum_{\substack{j \in \mathbb{M}_{k+1}(\zeta) \\ D_{k}\left(\varphi_{j}, \zeta\right)=\mathbf{d}}} c_{j}=0 .
$$

Suppose now $\mathbf{d}=\left(d_{0}, d_{1}, \ldots, d_{k}\right)$. There are a finite number of elements in $\mathcal{D}_{k+1}(\zeta)$ having the form $\left(d_{0}, d_{1}, \ldots, d_{k}, *\right)$, call them $\mathbf{d}_{i}=\left(d_{0}, d_{1}, \ldots, d_{k}, d_{k+1}^{i}\right), i=1$, $2, \ldots, r$. Then

$$
\sum_{\substack{j \in \mathbb{M}_{k+1}(\zeta) \\ D_{k}\left(\varphi_{j}, \zeta\right)=\mathbf{d}}} c_{j}=\sum_{i=1}^{r}\left\{\sum_{\substack{j \in \mathbb{M}_{k+1}(\zeta) \\ D_{k+1}\left(\varphi_{j}, \zeta\right)=\mathbf{d}_{i}}} c_{j}\right\}
$$


and each of the $r$ summands on the right vanishes, again by Eqn. (5.5). Therefore (ii) holds.

Now assume that (ii) holds. We consider the case $\beta=1$. The set $F$ is finite, say $F=\left\{\zeta_{1}, \ldots, \zeta_{s}\right\}$. Let $U_{1}, \ldots, U_{s}$ be disjoint arcs whose union is $\partial D$ and for which the interior of $U_{i}$ contains $\zeta_{i}$ for $i=1, \ldots, s$. Clearly,

$$
\sum_{j=1}^{n} c_{j} C_{\varphi_{j}}=\sum_{i=1}^{s} M_{\chi_{U_{i}}}\left(\sum_{j=1}^{n} c_{j} C_{\varphi_{j}}\right) .
$$

Taking $U$ to be any $U_{i}$ and $\zeta=\zeta_{i}$, it is enough to show that the operator

$$
M_{\chi_{U}}\left(\sum_{j=1}^{n} c_{j} C_{\varphi_{j}}\right)
$$

considered as acting from $H^{2}$ to $L^{2}$, is compact. This operator can be written as

$$
\sum_{\zeta \notin F\left(\varphi_{j}\right)} c_{j} M_{\chi_{U}} C_{\varphi_{j}}+\sum_{\zeta \in F\left(\varphi_{j}\right)} c_{j} M_{\chi_{U}} C_{\varphi_{j}} .
$$

If $F\left(\varphi_{j}\right)$ does not contain $\zeta$, then $\chi_{U}$ is identically zero in a neighborhood of $F\left(\varphi_{j}\right)$, and we see from Theorem 3.1 that $M_{\chi_{U}} C_{\varphi_{j}}$ is compact. Thus the first sum in the expression (5.6) is a compact operator.

Now consider the second sum, which can be rewritten as

$$
\sum_{m \geq 1}\left\{\sum_{j \in \mathbb{N}_{2 m}(\zeta)} c_{j} M_{\chi_{U}} C_{\varphi_{j}}\right\}
$$

the sum over $m$ is of course finite since $\mathbb{N}_{2 m}(\zeta)$ is empty for $m$ large enough. For non-empty $\mathbb{N}_{2 m}(\zeta)$,

$$
\sum_{j \in \mathbb{N}_{2 m}(\zeta)} c_{j} M_{\chi_{U}} C_{\varphi_{j}}=\sum_{\mathbf{d} \in \mathcal{E}_{2 m}(\zeta)}\left\{\sum_{\substack{j \in \mathbb{N}_{2 m}(\zeta) \\ D_{2 m}\left(\varphi_{j}, \zeta\right)=\mathbf{d}}} c_{j} M_{\chi_{U}} C_{\varphi_{j}}\right\} .
$$

If $\mathbf{d}$ is in $\mathcal{E}_{2 m}(\zeta), j$ and $\ell$ are in $\mathbb{N}_{2 m}(\zeta)$ and $D_{2 m}\left(\varphi_{j}, \zeta\right)=D_{2 m}\left(\varphi_{\ell}, \zeta\right)=\mathbf{d}$, Proposition $5.10(\mathrm{c})$ tells us that

$$
\left|\frac{\varphi_{j}\left(e^{i \theta}\right)-\varphi_{\ell}\left(e^{i \theta}\right)}{1-\overline{\varphi_{j}\left(e^{i \theta}\right)} \varphi_{\ell}\left(e^{i \theta}\right)}\right| \rightarrow 0 \text { as } e^{i \theta} \rightarrow \zeta .
$$

It follows from Theorem 4.4 that $M_{\chi_{U}}\left(C_{\varphi_{j}}-C_{\varphi_{\ell}}\right)$ is compact. Fix such $\ell$ and write $T=M_{\chi_{U}} C_{\varphi_{\ell}}$. Then for each $j$ in $\mathbb{N}_{2 m}(\zeta)$ with $D_{2 m}\left(\varphi_{j}, \zeta\right)=\mathbf{d}$, there is a compact operator $K_{j}$ with $M_{\chi_{U}} C_{\varphi_{j}}=T+K_{j}$. Thus

$$
\sum_{\substack{j \in \mathbb{N}_{2 m}(\zeta) \\ D_{2 m}\left(\varphi_{j}, \zeta\right)=\mathbf{d}}} c_{j} M_{\chi_{U}} C_{\varphi_{j}}=\left(\sum_{\substack{j \in \mathbb{N}_{2 m}(\zeta) \\ D_{2 m}\left(\varphi_{j}, \zeta\right)=\mathbf{d}}} c_{j}\right) T+\sum_{\substack{j \in \mathbb{N}_{2 m}(\zeta) \\ D_{2 m}\left(\varphi_{j}, \zeta\right)=\mathbf{d}}} c_{j} K_{j} .
$$

The coefficient of $T$ vanishes by the hypothesis (ii), and we are left with a compact operator, verifying (i). The proof for the case $\beta>1$ is similar, with Theorem $\mathrm{C}$ playing the role of Theorem 4.4, the sets $U_{i}$ taken to lie in $D$, and Proposition 5.10 (c) replaced by the assertion that if $2 m=2 n$ and $D_{2 m}(\varphi, \zeta)=D_{2 m}(\psi, \zeta)$, 
then $\rho(z) \rightarrow 0$ as $z \rightarrow \zeta$ unrestrictedly in $D$, an implication easily established by calculations in the proofs of Lemma 5.6 and Proposition 5.10.

Remark 5.14. It is sometimes convenient to rephrase condition (ii) in Theorem 5.13. With notation as in the statement, fix $\zeta$ in $F$, an even natural number $k$ and a vector $\mathbf{d}$ in $\mathcal{E}_{k}(\zeta)$. We define the vector $\mathbf{x}(\zeta, k, \mathbf{d})=\left(a_{1}, \ldots, a_{n}\right)$ in $\mathbb{C}^{n}$, where $a_{j}=1$ if $j$ is in $\mathbb{N}_{k}(\zeta)$ and $D_{k}\left(\varphi_{j}, \zeta\right)=\mathbf{d}$, while $a_{j}=0$ otherwise. Let $\mathcal{M}=\mathcal{M}\left(\varphi_{1}, \ldots, \varphi_{n}\right)$ denote the linear span in $\mathbb{C}^{n}$ of all such vectors $\mathbf{x}(\zeta, k, \mathbf{d})$. Clearly, condition (ii) in Theorem 5.13 is equivalent to

$$
\left(c_{1}, \ldots, c_{n}\right) \in \mathcal{M}^{\perp}
$$

Put another way, if we define a linear transformation $\mathbb{A}: \mathbb{C}^{n} \rightarrow \mathcal{B}\left(\mathcal{D}_{\beta}\right) / \mathcal{K}$ by

$$
\mathbb{A}\left(c_{1}, \ldots, c_{n}\right)=\left[c_{1} C_{\varphi_{1}}+\cdots+c_{n} C_{\varphi_{n}}\right],
$$

where $[B]$ denotes the coset of the operator $B$, then the content of Theorem 5.13 is that ker $\mathbb{A}=\mathcal{M}^{\perp}$. This yields the immediate:

Corollary 5.15. Fix $\varphi_{1}, \ldots, \varphi_{n}$ in $\mathcal{S}$. Then the vector subspace of $\mathcal{B}\left(\mathcal{D}_{\beta}\right) / \mathcal{K}$ spanned by the cosets $\left[C_{\varphi_{1}}\right], \ldots,\left[C_{\varphi_{n}}\right]$ has the same dimension as the subspace $\mathcal{M}\left(\varphi_{1}, \ldots, \varphi_{n}\right)$ of $\mathbb{C}^{n}$. In particular, these cosets are linearly independent if and only if $\mathcal{M}\left(\varphi_{1}, \ldots, \varphi_{n}\right)=\mathbb{C}^{n}$.

For maps $\varphi$ in $\mathcal{S}$ with order of contact uniformly two, the linear fractional selfmaps of $D$ play a special role. Let us write $\mathcal{S}(2)$ for the collection of those $\varphi$ in $\mathcal{S}$ which have order of contact two at each point of $F(\varphi)$. Further, we denote by $\mathcal{L}$ the collection of linear fractional self-maps $\varphi$ of $D$ which are not automorphisms but which have $\|\varphi\|_{\infty}=1$. We note that any linear fractional map $\psi$ is determined by its second-order data $D_{2}\left(\psi, z_{0}\right)=\left(\psi\left(z_{0}\right), \psi^{\prime}\left(z_{0}\right), \psi^{\prime \prime}\left(z_{0}\right)\right)$ at any point $z_{0}$ of analyticity. Suppose now that $\varphi$ is in $\mathcal{S}(2)$ and $\zeta_{0}$ is a point in $F(\varphi)$. Let $\varphi_{0}$ be the unique linear fractional map with $D_{2}\left(\varphi_{0}, \zeta_{0}\right)=D_{2}\left(\varphi, \zeta_{0}\right)$. Since the curvature of the parametric curve $\varphi\left(e^{i \theta}\right)$ is determined by second-order data, the circle $\varphi_{0}(\partial D)$ is necessarily the osculating circle of this curve at the point $\varphi\left(\zeta_{0}\right)$. Thus $\varphi_{0}$ maps $D$ to $D$ and lies in $\mathcal{L}$. The following result was established by the second author on the weighted Bergman spaces [16] here we extend it to $H^{2}$.

Corollary 5.16. Let $\varphi$ be in $\mathcal{S}(2)$ with $F(\varphi)=\left\{\zeta_{1}, \ldots, \zeta_{r}\right\}$. For $i=1, \ldots, r$ let $\varphi_{i}$ be the unique linear fractional map with $D_{2}\left(\varphi_{i}, \zeta_{i}\right)=D_{2}\left(\varphi, \zeta_{i}\right)$. Then

$$
C_{\varphi} \equiv C_{\varphi_{1}}+\cdots+C_{\varphi_{r}}(\bmod \mathcal{K})
$$

where the operators act on $H^{2}$.

Proof. This is immediate from Theorem 5.13 applied to the linear combination

$$
C_{\varphi}-C_{\varphi_{1}}-C_{\varphi_{2}}-\cdots-C_{\varphi_{r}}
$$

Let us write $\mathcal{V}_{2}$ for the vector subspace of $\mathcal{B}\left(\mathcal{D}_{\beta}\right) / \mathcal{K}$ spanned by the cosets $\left[C_{\varphi}\right]$ with $\varphi$ in $\mathcal{S}(2)$. According to Corollary 5.16, $\left\{\left[C_{\varphi}\right]: \varphi \in \mathcal{L}\right\}$ is a spanning subset of $\mathcal{V}_{2}$. That it is linearly independent as well follows immediately from Theorem 5.13, which gives the following:

Corollary 5.17. $\left\{\left[C_{\varphi}\right]: \varphi \in \mathcal{L}\right\}$ is a basis for $\mathcal{V}_{2}$. 
In Theorem 5.13, the proof that (ii) implies (i) uses the local compact difference Theorem 4.4 to locally "match up" certain pairs $C_{\varphi_{j}}$ and $C_{\varphi_{\ell}}$ by local data. As one changes the locality or local data, the pairs can change. However, the method suggests that with some minimality hypothesis, the coefficients in a linear equation

$$
c_{1} C_{\varphi_{1}}+\cdots+c_{n} C_{\varphi_{n}} \equiv 0(\bmod \mathcal{K})
$$

should be integers up to a common scalar factor. The next result makes this precise.

Corollary 5.18. Suppose that $\varphi, \varphi_{1}, \ldots, \varphi_{n}$ lie in $\mathcal{S}$ and assume that $\left[C_{\varphi_{1}}\right], \ldots$, $\left[C_{\varphi_{n}}\right]$ are linearly independent in $\mathcal{B}\left(\mathcal{D}_{\beta}\right) / \mathcal{K}$. If

$$
C_{\varphi} \equiv c_{1} C_{\varphi_{1}}+\cdots+c_{n} C_{\varphi_{n}}(\bmod \mathcal{K})
$$

then $c_{1}, \ldots, c_{n}$ are rational numbers.

Proof. Let us write $\psi_{1}=\varphi$ and $\psi_{k}=\varphi_{k-1}, b_{1}=-1$ and $b_{k}=c_{k-1}$ for $k=$ $2,3, \ldots, n+1$, so that the equation $\bmod \mathcal{K}$ in the statement becomes

$$
b_{1} C_{\psi_{1}}+b_{2} C_{\psi_{2}}+\cdots+b_{n+1} C_{\psi_{n+1}} \equiv 0(\bmod \mathcal{K}) .
$$

Linear independence of $\left[C_{\psi_{2}}\right], \ldots,\left[C_{\psi_{n+1}}\right]$ implies that $b_{1}=-1$ uniquely determines $b_{2}, \ldots, b_{n+1}$. Thus according to Remark $5.14, \mathcal{M}=\mathcal{M}\left(\psi_{1}, \ldots, \psi_{n}\right)$ has codimension one in $\mathbb{C}^{n+1}$. From the vectors $\mathbf{x}(\zeta, k, \mathbf{d})$ which $\operatorname{span} \mathcal{M}$, select a basis $\mathbf{x}_{1}, \ldots, \mathbf{x}_{n}$ for $\mathcal{M}$; the coordinates of each $\mathbf{x}_{i}$ are zeros and ones. On applying the Gram-Schmidt process to $\left\{\mathbf{x}_{1}, \ldots, \mathbf{x}_{n}\right\}$, we obtain an orthonormal basis for $\mathcal{M}$ of the form $\mathbf{z}_{i} /\left\|\mathbf{z}_{i}\right\|$, $i=1,2, \ldots, n$, where each $\mathbf{z}_{i}$ has rational coefficients. Let $P$ denote the orthogonal projection of $\mathbb{C}^{n+1}$ onto $\mathcal{M}$ and write $\mathbf{e}_{1}, \mathbf{e}_{2}, \ldots, \mathbf{e}_{n+1}$ for the standard basis vectors in $\mathbb{C}^{n+1}$. For some $j,(I-P) \mathbf{e}_{j}$ is non-zero, and clearly

$$
(I-P) \mathbf{e}_{j}=\mathbf{e}_{j}-\sum_{i=1}^{n} \frac{\left\langle\mathbf{e}_{j}, \mathbf{z}_{i}\right\rangle}{\left\|\mathbf{z}_{i}\right\|^{2}} \mathbf{z}_{i}
$$

is a vector $\left(r_{1}, r_{2}, \ldots, r_{n+1}\right)$ with obviously rational coefficients. Since $\mathcal{M}^{\perp}$ is onedimensional, $\left(b_{1}, \ldots, b_{n+1}\right)=c\left(r_{1}, \ldots, r_{n+1}\right)$ for some complex number $c$. Since $c r_{1}=b_{1}=-1, c$ is rational so that $b_{2}, \ldots, b_{n+1}$ are rational as well.

Finally, we note that certain weighted composition operators enjoy a decomposition analogous to that in Corollary 5.16, with the sum replaced by a linear combination.

Proposition 5.19. Let $\varphi$ be in $\mathcal{S}(2)$ with $F(\varphi)=\left\{\zeta_{1}, \ldots, \zeta_{r}\right\}$ and suppose that $\varphi_{i}$, $i=1, \ldots, r$, are linear fractional maps related to $\varphi$ as in Corollary 5.16. If $w$ is a bounded measurable function on $\partial D$ which is continuous at each point of $F(\varphi)$, then

$$
M_{w} C_{\varphi} \equiv w\left(\zeta_{1}\right) C_{\varphi_{1}}+\cdots+w\left(\zeta_{r}\right) C_{\varphi_{r}}(\bmod \mathcal{K}),
$$

where the operators are considered as mapping $H^{2}$ to $L^{2}$.

Proof. Choose pairwise disjoint arcs $I_{1}, \ldots, I_{r}$ with union $\partial \mathbb{D}$ and the interior of $I_{j}$ containing $\zeta_{j}$ for $j=1, \ldots, r$. We write $\chi_{j}$ for $\chi_{I_{j}}$. We have

$$
M_{w} C_{\varphi}=\sum_{j=1}^{r}\left[M_{\left(w-w\left(\zeta_{j}\right)\right) \chi_{j}} C_{\varphi}+w\left(\zeta_{j}\right) M_{\chi_{j}} C_{\varphi}\right] .
$$


The first term in each summand on the right is compact by Theorem 3.1. Moreover the operators

$$
M_{\chi_{j}}\left(C_{\varphi}-C_{\varphi_{j}}\right) \text { and } M_{\left(1-\chi_{j}\right)} C_{\varphi_{j}}
$$

are compact, the first by Theorem 4.4 and Proposition 5.10(c) and the second by Theorem 3.1; the proposition follows.

Note that left-multiplying all operators in the proposition by the orthogonal projection $P$ of $L^{2}$ onto $H^{2}$ gives an alternate statement in which the multiplication operator $M_{w}$ is replaced by the corresponding Toeplitz operator $T_{w}=P M_{w} \mid H^{2}$, and all operators act from $H^{2}$ to $H^{2}$.

\section{ArC-COnNeCtedness in $\mathcal{C}\left(\mathcal{S}_{0}\right)$}

In [26, Shapiro and Sundberg posed the following interesting question: What is the relationship between the conditions

(a) $C_{\varphi}-C_{\psi}$ is compact;

(b) $C_{\varphi}$ and $C_{\psi}$ lie in the same component of the topological space of composition operators on $\mathcal{D}_{\beta}$ ?

The second author and Toews [17 proposed the scheme discussed in Section 2.6 and used it to give examples of composition operators satisfying (b) but not (a). Bourdon 3 presented analogous examples for linear fractional maps, showing that for $\varphi$ and $\psi$ in the class $\mathcal{L}$ (see Section 5.5), (a) corresponds to equal second-order data at the (common) point of contact with $\partial D$ whereas equal first-order data characterizes (b). The second author [16] proved the corresponding result on the Bergman spaces for $\varphi$ and $\psi$ in the class $\mathcal{S}_{0} \cap \mathcal{S}(2)$ where $\mathcal{S}_{0}$ is as defined below. Here we extend this result to $H^{2}$ and to higher orders of contact. First we record a general sufficient condition for (b), proved in [16] for the Bergman spaces.

Proposition 6.1. Let us write $\varphi_{t}=t \varphi+(1-t) \psi, 0 \leq t \leq 1$, where $\varphi$ and $\psi$ are

analytic self-maps of $D$. If $\rho=\left|\frac{\varphi-\psi}{1-\bar{\varphi} \psi}\right|$ is essentially bounded away from one on $\partial D$, then there is a constant $B$ such that

$$
\left\|C_{\varphi_{s}}-C_{\varphi_{r}}\right\|_{\mathcal{B}\left(H^{2}\right)} \leq B|s-r|, \quad 0 \leq r<s \leq 1 .
$$

The proposition follows immediately from Theorem 2.2 and Lemma 4.1 with $G$ taken to be the appropriate set of full measure.

We write $\mathcal{S}_{0}$ for the collection of those $\varphi$ in $\mathcal{S}$ such that $\left\|\chi_{\partial D \backslash U} \varphi\right\|_{\infty}<1$ for every open subset $U$ of $\partial D$ containing $F(\varphi)$. Such a $\varphi$ is allowed to make contact with $\partial D$ only at points of $F(\varphi)$. We also write $\mathcal{C}\left(\mathcal{S}_{0}\right)$ for the set of those $C_{\varphi}$ with $\varphi$ in $\mathcal{S}_{0}$.

Theorem 6.2. Suppose that $\varphi$ and $\psi$ lie in $\mathcal{S}_{0}$ with respective orders of contact $2 m(\zeta)$ and $2 n(\zeta)$ at each $\zeta$ in (respectively) $F(\varphi)$ and $F(\psi)$. Let $\varphi_{t}=t \varphi+(1-t) \psi$, $0 \leq t \leq 1$. Then if $\beta \geq 1$, the following are equivalent:

(i) $C_{\varphi}$ and $C_{\psi}$ lie in the same component of the space $\mathcal{C}\left(\mathcal{S}_{0}\right)$ equipped with the norm topology of $\mathcal{B}\left(\mathcal{D}_{\beta}\right)$.

(ii) There is a positive constant $B$ such that

$$
\left\|C_{\varphi_{s}}-C_{\varphi_{r}}\right\| \leq B|s-r|, \quad 0 \leq r<s \leq 1 .
$$

(iii) $F(\varphi)=F(\psi)$ (call this set $F$ ), and for each $\zeta$ in $F, 2 m(\zeta)=2 n(\zeta)$ and $D_{2 m(\zeta)-1}(\varphi, \zeta)=D_{2 m(\zeta)-1}(\psi, \zeta)$. 
Proof. First consider the $H^{2}$ case. Suppose that (iii) holds and write $\rho=\left|\frac{\varphi-\psi}{1-\bar{\varphi} \psi}\right|$ as usual. If $\zeta$ is in $F$, it is clear from Proposition 5.10 that $\lim _{e^{i \theta} \rightarrow \zeta} \rho\left(e^{i \theta}\right)<1$. Thus there is an open set $U$ in $\partial D$ containing $F$ such that $\left\|\chi_{U} \rho\right\|_{\infty}<1$. Since $\varphi$ and $\psi$ lie in $\mathcal{S}_{0},\left\|\chi_{\partial D \backslash U} \rho\right\|_{\infty}<1$ as well, and (ii) follows from Proposition 6.1. For the Bergman space version of this implication, replace $U$ by an appropriate subset of $D$ and use the Bergman space analogue of Proposition 6.1 in [16], together with the following replacement for Proposition 5.10(b), a fact easily established by calculations in the proofs of Lemma 5.6 and Proposition 5.10: If $\varphi$ and $\psi$ have sufficient data and common order of contact $2 m$ at $\zeta$ in $\partial D$, and if $D_{2 m-1}(\varphi, \zeta)=$ $D_{2 m-1}(\psi, \zeta)$, then

$$
\limsup _{z \rightarrow \zeta} \rho(z)<1
$$

the limit superior being taken unrestrictedly in $D$. Clearly (ii) implies (i) on any $\mathcal{D}_{\beta}$. Finally, we can use the topological argument of Theorem 2.4 in [13], with Corollary 5.8 playing the role of Theorem 2.2 in [13, to show that (i) implies (iii).

The last theorem and Corollary 5.12 show that the phenomenon (a) $\Rightarrow$ (b) (and not conversely) persists more broadly. Note, however, that by passing from the class $\mathcal{S}$ to $\mathcal{S}_{0}$, we have eliminated maps $\varphi$ which touch the unit circle outside of $E(\varphi)$. Such points of contact are immaterial for condition (a); we do not know whether they matter for (b).

\section{REFERENCES}

[1] A. Aleksandrov, Multiplicity of boundary values of inner functions, Izv. Akad. Nauk Armyan. SSR Ser. Mat. 22 (1987), no. 5, 490-503, 515. MR0931885 (89e:30058)

[2] E. Berkson, Composition operators isolated in the uniform operator topology, Proc. Amer. Math. Soc. 81 (1981), 230-232. MR0593463 (82f:47039)

[3] P. S. Bourdon, Components of linear-fractional composition operators, J. Math. Anal. Appl. 279 (2003), 228-245. MR 1970503 (2004c:47047)

[4] P. S. Bourdon, D. Levi, S. K. Narayan, and J. H. Shapiro, Which linear fractional composition operators are essentially normal? J. Math. Anal. Appl. 280 (2003), 30-53. MR1972190 (2003m:47042)

[5] J. A. Cima and A. L. Matheson, Essential norms of composition operators and Aleksandrov measures, Pacific J. Math. 179 (1997), 59-64. MR1452525 (98e:47047)

[6] D. N. Clark, One-dimensional perturbations of restricted shifts, J. D'Analyse Math. 25 (1972), 169-191. MR0301534(46:692)

[7] C. C. Cowen, Composition operators on $H^{2}$, J. Operator Theory 9 (1983), 77-106. MR0695941 (84d:47038)

[8] C. C. Cowen and B. D. MacCluer, Composition Operators on Spaces of Analytic Functions, Studies in Advanced Mathematics, CRC Press, Boca Raton, FL, 1995. MR:1397026 (97i:47056)

[9] P. L. Duren, Theory of $H^{p}$ Spaces, Academic Press, New York, 1970. MR0268655 (42:3552)

[10] P. Gorkin and R. Mortini, Norms and essential norms of linear combinations of endomorphisms, Trans. Amer. Math. Soc. 358 (2006), no. 2, 553-571. MR2177030 (2006h:47055)

[11] P. R. Halmos, Measure Theory, Van Nostrand, New York, 1950. MR0033869 (11:504d)

[12] K. Hoffman, Banach Spaces of Analytic Functions, Prentice Hall, Englewood Cliffs, NJ, 1962. MR0133008 (24:A2844)

[13] B. D. MacCluer, Components in the space of composition operators, Integral Equations and Operator Theory 12 (1989), 725-738. MR1009027 (91b:47070)

[14] B. D. MacCluer, S. Ohno, and R. Zhao, Topological structure of the space of composition operators on $H^{\infty}$, Integral Equations and Operator Theory 40 (2001), 481-494. MR1839472 (2002d:47039) 
[15] B. D. MacCluer and J. H. Shapiro, Angular derivatives and compact composition operators on the Hardy and Bergman spaces, Canadian J. Math. 38 (1986), 878-906. MR0854144 (87h:47048)

[16] J. Moorhouse, Compact differences of composition operators, J. Functional Analysis 219 (2005), 70-92. MR.2108359(2005i:47037)

[17] J. Moorhouse and C. Toews, Differences of composition operators, Trends in Banach Spaces and Operator Theory (Memphis, TN, 2001), 207-213, Contemp. Math. 321, Amer. Math. Soc., Providence, RI, 2003. MR.1978818 (2004b:47046)

[18] N. K. Nikolski, Operators, Functions, and Systems: An Easy Reading, Volume 1: Hardy, Hankel and Toeplitz, Mathematical Surveys and Monographs, 92, Amer. Math. Soc., Providence, RI, 2002. MR1864396 (2003i:47001a)

[19] P. J. Nieminen and E. Saksman, On the compactness of the difference of composition operators, J. Math. Anal. Appl. 298 (2004), 501-522. MR2086972(2005e:30065)

[20] A. G. Poltoratski, Boundary behavior of pseudocontinuable functions (Russian), Algebra $i$ Analiz 5 (1993), 189-210; translation in St. Petersburg Math. J. 5 (1994), 389-406. MR.1223178 (94k:30090)

[21] S. Saitoh, Integral Transforms, Reproducing Kernels and their Applications, Pitman Research Notes in Mathematics, 369, Longman, Harlow, 1997. MR1478165 (98k:46041)

[22] D. E. Sarason, Composition operators as integral operators, Analysis and Partial Differential Equations, C. Sadosky (ed.), 545-565, Marcel-Dekker, New York, 1990. MR1044808 (92a:47040)

[23] D. E. Sarason, Sub-Hardy Hilbert Spaces in the Unit Disk, University of Arkansas Lecture Notes in the Mathematical Sciences, Volume 10, John Wiley and Sons, New York, 1994. MR:1289670 (96k:46039)

[24] J. E. Shapiro, Aleksandrov measures used in essential norm inequalities for composition operators, J. Operator Theory 40 (1998), 133-146. MR.1642538 (99i:47062)

[25] J. H. Shapiro, The essential norm of a composition operator, Annals Math. 125 (1987), 375404. MR0881273 (88c:47058)

[26] J. H. Shapiro and C. Sundberg, Isolation amongst the composition operators, Pacific J. Math. 145 (1990), 117-152. MR1066401 (92g:47041)

[27] J. H. Shapiro and C. Sundberg, Compact composition operators on $L^{1}$, Proc. Amer. Math. Soc. 108 (1990), 443-449. MR0994787 (90d:47035)

[28] J. H. Shapiro and P. D. Taylor, Compact, nuclear and Hilbert-Schmidt composition operators on $H^{2}$, Indiana Univ. Math. J. 23 (1973), 471-496. MR0326472 (48:4816)

Department of Mathematics, University of Virginia, Charlottesville, Virginia 22904

E-mail address: tlk8q@virginia.edu

Department of Mathematics, Colgate University, Hamilton, New York 11346

E-mail address: jmoorhouse@mail-colgate.edu 